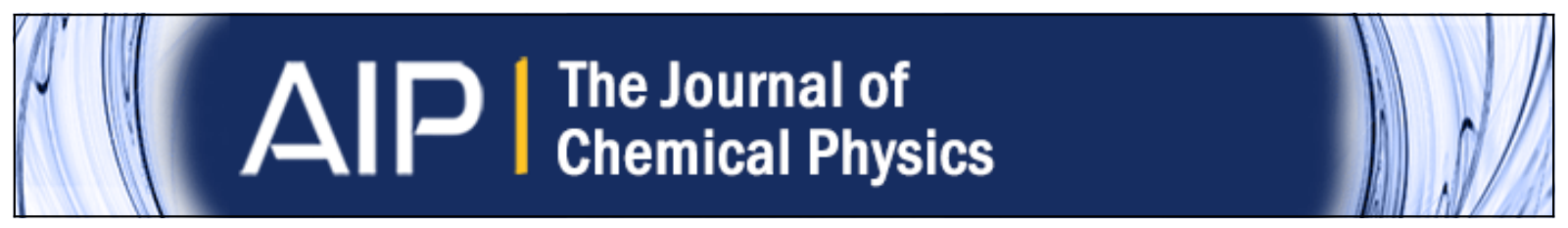

The isotropic-nematic and nematic-nematic phase transition of binary mixtures of tangent hard-sphere chain fluids: An analytical equation of state

Thijs van Westen, Thijs J. H. Vlugt, and Joachim Gross

Citation: The Journal of Chemical Physics 140, 034504 (2014); doi: 10.1063/1.4860980

View online: http://dx.doi.org/10.1063/1.4860980

View Table of Contents: http://scitation.aip.org/content/aip/journal/jcp/140/3?ver=pdfcov

Published by the AIP Publishing

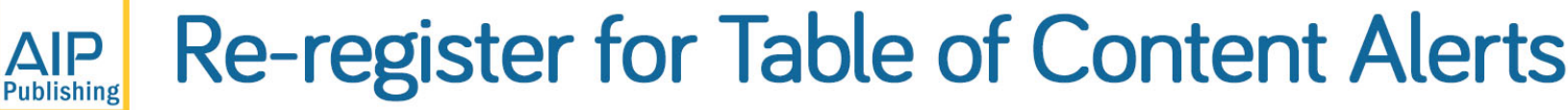




\title{
The isotropic-nematic and nematic-nematic phase transition of binary mixtures of tangent hard-sphere chain fluids: An analytical equation of state
}

\author{
Thijs van Westen, ${ }^{1}$ Thijs J. H. Vlugt, ${ }^{1}$ and Joachim Gross ${ }^{2, a)}$ \\ ${ }^{1}$ Process and Energy Laboratory, Delft University of Technology, Leeghwaterstraat 44, 2628 CA Delft, \\ The Netherlands \\ ${ }^{2}$ Institut für Thermodynamik und Thermische Verfahrenstechnik, Universität Stuttgart, Pfaffenwaldring 9, \\ 70569 Stuttgart, Germany
}

(Received 31 October 2013; accepted 19 December 2013; published online 16 January 2014)

\begin{abstract}
An analytical equation of state (EoS) is derived to describe the isotropic (I) and nematic (N) phase of linear- and partially flexible tangent hard-sphere chain fluids and their mixtures. The EoS is based on an extension of Onsager's second virial theory that was developed in our previous work [T. van Westen, B. Oyarzún, T. J. H. Vlugt, and J. Gross, J. Chem. Phys. 139, 034505 (2013)]. Higher virial coefficients are calculated using a Vega-Lago rescaling procedure, which is hereby generalized to mixtures. The EoS is used to study (1) the effect of length bidispersity on the I-N and N-N phase behavior of binary linear tangent hard-sphere chain fluid mixtures, (2) the effect of partial molecular flexibility on the binary phase diagram, and (3) the solubility of hard-sphere solutes in I- and N tangent hard-sphere chain fluids. By changing the length bidispersity, two types of phase diagrams were found. The first type is characterized by an I-N region at low pressure and a N-N demixed region at higher pressure that starts from an I-N-N triphase equilibrium. The second type does not show the I-N-N equilibrium. Instead, the N-N region starts from a lower critical point at a pressure above the I-N region. The results for the I-N region are in excellent agreement with the results from molecular simulations. It is shown that the N-N demixing is driven both by orientational and configurational/excluded volume entropy. By making the chains partially flexible, it is shown that the driving force resulting from the configurational entropy is reduced (due to a less anisotropic pairexcluded volume), resulting in a shift of the N-N demixed region to higher pressure. Compared to linear chains, no topological differences in the phase diagram were found. We show that the solubility of hard-sphere solutes decreases across the I-N phase transition. Furthermore, it is shown that by using a liquid crystal mixture as the solvent, the solubility difference can by maximized by tuning the composition. Theoretical results for the Henry's law constant of the hard-sphere solute are in good agreement with the results from molecular simulation. (C) 2014 AIP Publishing LLC. [http://dx.doi.org/10.1063/1.4860980]
\end{abstract}

\section{INTRODUCTION}

Mixtures of liquid crystals (LCs) with other LCs, polymers or non-LC additives are commonly employed to tailor the properties of liquid crystalline materials for specific tasks. ${ }^{1,2}$ Some examples are the addition of chiral dopants to achiral LCs to induce supra-molecular chirality for LC displays, ${ }^{2-4}$ the mixing of LCs to enhance the range of stability of a nematic phase, ${ }^{1}$ the mixing of LCs and polymers for improved polymer processing, ${ }^{5}$ and the addition of LCs to membranes for improved transport properties. ${ }^{5}$ Also, mixing LCs of different molecular architecture is known to produce very different phase behavior compared to the pure components. $2,6,7$

The theoretical description of LCs dates back to the work of Onsager. $^{8,9}$ In this seminal work, the isotropic-nematic (I-N) phase transition was explained as a consequence of a competition between an orientational entropy, favoring the I

\footnotetext{
a) Author to whom correspondence should be addressed. Electronic mail: gross@itt.uni-stuttgart.de
}

(isotropic, disordered) state, and a configurational entropy, favoring the $\mathrm{N}$ (nematic, orientationally ordered) state. Although Onsager performed calculations for pure components only, he argued that for mixtures of (athermal) LCs of different length, the longer molecules would preferentially partition in the $\mathrm{N}$ phase, leading to a fractionation of components between the coexisting phases (fractionation effect). Since then, different experimental studies have confirmed this hypothesis. ${ }^{10-12}$ The first theoretical confirmation is due to Flory and Abe, ${ }^{13}$ who studied a binary mixture of rod-like polymers in the lattice approximation. Later, Lekkerkerker et al. extended Onsager's formalism to mixtures and confirmed his predictions from a continuum fluid approach. ${ }^{14}$ From that point, numerous theoretical ${ }^{15-23}$ and some molecular simulation ${ }^{18,24-26}$ studies have been performed on the subject, showing interesting phase behavior such as the fractionation effect, ${ }^{15,17-24}$ demixing (N-N, ${ }^{16,20,21,23,25-27} \mathrm{I}-\mathrm{I},{ }^{17,20,28}$ and smectic-smectic $\left.{ }^{19,21}\right)$, re-entrant phenomena, ${ }^{15-17,20,23}$ and density inversion ${ }^{27}$ (isotropic phase denser than LC phase). Many of these phenomena have also been observed experimentally. ${ }^{12,29-31}$ 
In the present work, we study the I-N phase behavior and N-N demixing behavior of binary mixtures of tangent hard-sphere chain fluids within a Vega-Lago rescaled Onsager theory. ${ }^{32}$ We herewith extend our previous work on pure component systems. ${ }^{33}$ For an overview of related work on pure component systems, the reader is referred to Refs. 34-49. Due to the combined use of an analytical approximation for the pair-excluded volume ${ }^{50}$ and the Onsager Trial Function for the orientational distribution function (ODF), an analytical equation of state (EoS) is obtained (Sec. III). In particular, this analytical EoS is used to study the effect of changing the length ratio of two linear chains in a binary mixture (length bidispersity) on the I-N and N-N phase behavior (Sec. IV A). Furthermore, we investigate the effect of making one component of the binary mixture partially flexible (Sec. IV B). The partial flexibility is introduced by the rod-coil model. Finally, the solubility of hard spheres in linear and partially flexible tangent hard-sphere chain solvents is studied (Sec. IV C).

\section{MOLECULAR MODEL}

The molecular architecture of typical liquid crystal molecules of prolate type consists of a hard, rigid, anisotropic core with one or more (semi-)flexible tail-groups attached to its end(s).$^{51,52}$ To mimic this architecture, we assume a chain of $m$ tangent hard spheres of diameter $\sigma$, where one part of the chain is arranged in a linear conformation (referred to as "rod") while the other part is fully flexible (referred to as "coil"). This model will be referred to as rod-coil fluid. ${ }^{33,50,53}$ We employ a general $m-m_{R}$ notation to denote a rod-coil with a total number of $m$ segments and a number of $m_{R}$ segments in the rigid block. A completely linear chain is referred to as a linear $m$-mer.

As a measure for the partial flexibility of a molecule, a dimensionless rigidity parameter is introduced, which, following our previous work, ${ }^{33,50,53}$ is defined as the ratio of the total number of rigid bond-angles and the total number of bondangles in a molecule:

$$
\chi_{\mathrm{R}}=\left\{\begin{array}{ll}
\frac{m_{\mathrm{R}}-2}{m-2} & \text { for } m>2 \\
1 & \text { for } m \leq 2
\end{array} .\right.
$$

The rigidity parameter serves as an input for the excluded volume expression ${ }^{50}$ and isotropic equation of state ${ }^{53}$ used in this work. It varies conveniently from zero to unity between the completely flexible- and rigid chain limit, respectively.

\section{THEORY}

In our previous work, ${ }^{33}$ an Onsager-like Helmholtz energy functional was derived to describe the isotropic-nematic ordering transition in pure component systems of tangent hard-sphere chain molecules. The resulting Helmholtz energy functional could be expressed in terms of an orientational distribution function only, leading to a unified description of linear and partially flexible (rod-coil) tangent hard-sphere chain fluids. In the present work (Sec. III A), the developed formalism is extended to mixtures. To avoid repetition, only the main points needed for the extension are discussed. In
Sec. III B, details on the solution of the phase equilibrium are provided. Furthermore, in Sec. III C we use the Onsager Trial Function (OTF) approximation ${ }^{9,54}$ to derive analytical results for the Helmholtz energy of the nematic fluid mixture.

\section{A. Helmholtz energy functional}

For a canonical, multicomponent mixture (of $N_{C}$ components) of tangent hard-sphere chain molecules, the total reduced Helmholtz energy density can be written as the sum of an ideal and residual contribution, according to

$$
a[f(\boldsymbol{\omega})]=\frac{\beta A[f(\boldsymbol{\omega})]}{V}=a^{\mathrm{id}}[f(\boldsymbol{\omega})]+a^{\mathrm{res}}[f(\boldsymbol{\omega})] .
$$

Here, $\beta^{-1}=k T$ is the product of Boltzmann's constant $k$ with the absolute temperature $T, V$ is the volume of the system, and the vector $\omega$ is the orientation of a molecule's axis with respect to the director (average direction of all molecules in a phase). The probability density to find any molecule of a component $i$ in an orientation $\omega$ is defined by the orientational distribution function (ODF) $f_{i}(\boldsymbol{\omega})$, which is normalized as $\int f_{i}(\boldsymbol{\omega}) \mathrm{d} \boldsymbol{\omega}=1$. We use the shorthand notation $f(\boldsymbol{\omega})$ to denote the ODFs $f_{1}(\boldsymbol{\omega}), f_{2}(\boldsymbol{\omega}), \ldots, f_{N_{C}}(\boldsymbol{\omega})$ of all $N_{C}$ components in the mixture.

The ideal contribution can be written as the sum of an isotropic part and an anisotropic part (related to the orientational entropy) as

$$
a^{\mathrm{id}}[f(\boldsymbol{\omega})]=a_{\mathrm{iso}}^{\mathrm{id}}+a_{\mathrm{aniso}}^{\mathrm{id}}[f(\boldsymbol{\omega})],
$$

where

$$
\begin{gathered}
a_{\mathrm{iso}}^{\mathrm{id}}=\sum_{i}^{N_{C}} \rho_{i}\left[\ln \left(\frac{\rho_{i} \Lambda_{i}^{3}}{\Omega}\right)-1\right], \\
a_{\text {aniso }}^{\mathrm{id}}[f(\boldsymbol{\omega})]=\sum_{i}^{N_{C}} \rho_{i} \int f_{i}(\boldsymbol{\omega}) \ln \left[\Omega f_{i}(\boldsymbol{\omega})\right] \mathrm{d} \boldsymbol{\omega} .
\end{gathered}
$$

Here, $\rho_{i}=N_{i} / V$ is the number density of molecules of component $i, \Lambda_{i}^{3}$ is a generalized De Broglie volume (which for partially flexible molecules incorporates a contribution due to the internal configurational degrees of freedom of a molecule $^{33}$ ) and the factor $\Omega=\int \mathrm{d} \omega=4 \pi$ is a normalization constant which ensures that the anisotropic part vanishes for an isotropic distribution of molecular orientations (since $\left.f_{i, \text { iso }}(\boldsymbol{\omega})=1 / \Omega=1 / 4 \pi\right)$.

Analogous to our previous work, the Vega-Lago rescaling ${ }^{32}$ is used to describe the residual Helmholtz energy. In this approach, the higher virial coefficients of the nematic fluid are approximated by a mapping onto those of an isotropic fluid using a scaling of second virial coefficients. Upon introducing a second virial coefficient of the mixture $\bar{B}_{2}$, the Vega-Lago approach can be generalized to mixtures as

$$
a^{\mathrm{res}}[f(\boldsymbol{\omega})]=a_{\mathrm{iso}}^{\mathrm{res}} \frac{\bar{B}_{2}[f(\boldsymbol{\omega})]}{\bar{B}_{2, \text { iso }}} .
$$


Differentiation with respect to density results in the compressibility factor $Z=\beta P / \rho$, according to

$$
Z=1+Z_{\text {iso }}^{\text {res }} \frac{\bar{B}_{2}[f(\boldsymbol{\omega})]}{\bar{B}_{2, \text { iso }}} .
$$

For a more rigorous derivation of the above equation, the reader is referred to Appendix A. Clearly, a prerequisite for the application of the Vega-Lago approach is an accurate description of the thermodynamic properties of the isotropic phase. In the present work, the description of the isotropic phase is obtained from the LH-rc $\operatorname{EoS}^{53}$ (see Appendix B for details). In contrary to conventional EoS for hard-chain fluids, such as TPT $1^{55}$ and the LH EoS, ${ }^{56,57}$ the LH-rc EoS explicitly considers the effect of intramolecular flexibility, leading to an improved description of the isotropic compressibility factor of linear and partially flexible tangent hard-sphere chain fluids and their mixtures. Also, for the pure-component system, we have demonstrated that when combined with a Vega-Lago rescaling, the LH-rc EoS leads to an accurate description of the nematic fluid. ${ }^{33}$

The second virial coefficient of the mixture that is needed in Eqs. (6) and (7) is defined as a mole-fraction-weighted sum of the second virial coefficients over all possible pair interactions, ${ }^{58,59}$ according to

$$
\bar{B}_{2}[f(\boldsymbol{\omega})]=\sum_{i}^{N_{C}} \sum_{j}^{N_{C}} x_{i} x_{j} B_{2, i j}\left[f_{i}(\boldsymbol{\omega}), f_{j}(\boldsymbol{\omega}] .\right.
$$

For purely repulsive, rigid, cylindrically symmetric, hard-core molecules, the second virial coefficient of any two molecules 1 and 2 of type $i$ and $j$, respectively, can be written in terms of an orientational average of their pair-excluded volume, according to ${ }^{9}$

$$
\begin{aligned}
B_{2, i j}\left[f_{i}(\boldsymbol{\omega}), f_{j}(\boldsymbol{\omega})\right] & =\frac{1}{2} \iint V_{\mathrm{ex}, i j}(\gamma) f_{i}\left(\boldsymbol{\omega}_{1}\right) f_{j}\left(\boldsymbol{\omega}_{2}\right) \mathrm{d} \boldsymbol{\omega}_{1} \mathrm{~d} \boldsymbol{\omega}_{2} \\
& =\frac{1}{2}\left\langle\left. V_{\mathrm{ex}, i j}(\gamma)\right|_{\boldsymbol{\omega}_{1}, \boldsymbol{\omega}_{2}} .\right.
\end{aligned}
$$

Here, $\gamma$ is the angle between the major principal axes of the two molecules. For partially flexible molecules, the pairexcluded volume is not uniquely defined by the intermolecular angle $\gamma$ but also depends on the intramolecular configurations (bond- and torsion angles) of the two molecules. To apply the above equation to this type of molecules, we follow the method laid out in our previous work, ${ }^{33}$ and obtain $V_{\mathrm{ex}, i j}(\gamma)$ from a pre-averaging over all internal configurations. The required ensemble average is accurately described by the following analytical approximation: ${ }^{50}$

$$
V_{\mathrm{ex}, i j}(\gamma)=V_{\bar{m}_{i j}} \sum_{k=1}^{3} C_{k, i j}\left(\bar{m}_{i j}, \bar{\chi}_{\mathrm{R}, i j}\right) \sin ^{k-1}(\gamma) .
$$

Here, $V_{\bar{m}_{i j}}$ is the molecular volume of a chain of $\bar{m}_{i j}$ tangent hard spheres, where we have introduced an average chain length and rigidity parameter of two molecules of type $i$ and $j$ as

$$
\bar{m}_{i j}=\frac{m_{i}+m_{j}}{2},
$$

$$
\bar{\chi}_{\mathrm{R}, i j}=\frac{\chi_{\mathrm{R}, i}+\chi_{\mathrm{R}, j}}{2} .
$$

The coefficients $C_{1, i j}\left(\bar{m}_{i j}, \bar{\chi}_{\mathrm{R}, i j}\right), \quad C_{2, i j}\left(\bar{m}_{i j}, \bar{\chi}_{\mathrm{R}, i j}\right)$, and $C_{3, i j}\left(\bar{m}_{i j}, \bar{\chi}_{\mathrm{R}, i j}\right)$ were obtained by correlating MC data for the orientation-dependent pair-excluded volume of purecomponent rod-coil fluids ${ }^{50}$ For the functional form of these coefficients, the reader is referred to Appendix C. It is worthwhile to note that for linear chains, the above correlation for the pair-excluded volume reduces to the quasi-exact result of Williamson and Jackson, ${ }^{60}$ which we later extended to mixtures. ${ }^{50}$

The isotropic second virial coefficient of the individual pair interactions that is needed to calculate $\bar{B}_{2 \text {,iso }}$ in Eqs. (6) and (7) is obtained by inserting Eq. (10) in Eq. (9) and using $f_{i, \text { iso }}(\boldsymbol{\omega})=1 / \Omega$ (which follows from the normalization condition):

$$
\begin{aligned}
B_{2, \text { iso }, i j}= & V_{\bar{m}_{i j}}\left[\frac{C_{1, i j}\left(\bar{m}_{i j}, \bar{\chi}_{\mathrm{R}, i j}\right)}{2}\right. \\
& \left.+\frac{\pi C_{2, i j}\left(\bar{m}_{i j}, \bar{\chi}_{\mathrm{R}, i j}\right)}{8}+\frac{C_{3, i j}\left(\bar{m}_{i j}, \bar{\chi}_{\mathrm{R}, i j}\right)}{3}\right] .
\end{aligned}
$$

Equations (2)-(13) completely define our extension of the Onsager-Vega-Lago theory to mixtures of linear and/or (partially) flexible tangent hard-sphere chain fluids.

\section{B. Solving the phase equilibrium}

For the purely repulsive molecules considered in this work, the determination of phase equilibrium between two phases $\mathrm{A}$ and $\mathrm{B}$ follows from the equality of pressure $(P)$ and chemical potential of each component $i\left(\mu_{i}\right)$ in the coexisting phases

$$
\begin{gathered}
P^{\mathrm{A}}=P^{\mathrm{B}}, \\
\mu_{i}^{\mathrm{A}}=\mu_{i}^{\mathrm{B}} \quad \text { for } \quad i=1,2, \ldots, N_{C} .
\end{gathered}
$$

In terms of the Helmholtz-energy density $a=\beta A / V$, these can be obtained as

$$
\begin{gathered}
\beta P=-a+\sum_{i}^{N_{C}} \rho_{i}\left(\frac{\partial a\left[f_{\mathrm{eq}}(\boldsymbol{\omega})\right]}{\partial \rho_{i}}\right)_{T \rho_{j \neq i}}, \\
\beta \mu_{i}=\left(\frac{\partial a\left[f_{\mathrm{eq}}(\boldsymbol{\omega})\right]}{\partial \rho_{i}}\right)_{T \rho_{j \neq i}} .
\end{gathered}
$$

To calculate these quantities for a nematic phase, one first has to solve for the equilibrium ODFs which minimize the total Helmholtz energy. Although one can use a functional minimization for this, ${ }^{17,22,61}$ the numerical difficulties involved in such a method are quite severe. Instead, we choose the OTF approach and assume the ODF of a component $i$ can be approximated as ${ }^{9}$

$$
f_{i}(\boldsymbol{\omega}) \approx f_{\mathrm{OTF}, i}(\theta)=\frac{\alpha_{i} \cosh \left[\alpha_{i} \cos (\theta)\right]}{4 \pi \sinh \left(\alpha_{i}\right)} .
$$

Here, $\alpha_{i}$ is a variational parameter defining the degree of orientational order of a component $i . \theta$ is the polar angle of a molecule's axis with respect to the nematic director. Although the use of the OTF is approximate, recent work has shown that 
for many systems it leads to results that are similar in accuracy to those obtained from using a full numerical solution of the ODF. ${ }^{33,54}$

The merit of using the OTF is that the orientation dependence of the Helmholtz energy can be reduced solely to the $\alpha$ parameters (see Sec. III C for the details on this). Accordingly, the functional minimization can be reduced to a simpler parameter minimization, and the equilibrium orientational state of the system is obtained by simultaneously solving the following set of nonlinear equations for $i=1,2, \ldots, N_{C}$

$$
\left(\frac{\partial a(\boldsymbol{\alpha})}{\partial \alpha_{i}}\right)_{T \rho, \boldsymbol{\alpha}=\boldsymbol{\alpha}_{e q}}=0 .
$$

Here, $\boldsymbol{\alpha}$ is a vector containing the $\alpha_{i}$ of all $N_{C}$ components in the mixture. A modified Newton-Raphson method ${ }^{62}$ was used to solve this problem. The resulting $\boldsymbol{\alpha}_{\text {eq }}$ determine the equilibrium ODFs according to Eq. (18). Accordingly, the (partial) nematic order parameter of a component $i$ can be calculated from $^{9}$

$$
S_{2, i}=1-\frac{3 \operatorname{coth}\left(\alpha_{i}\right)}{\alpha_{i}}+\frac{3}{\alpha_{i}^{2}} .
$$

We choose to calculate the total nematic order parameter of the mixture as a simple mole-fraction-weighted sum of the partial nematic order parameters, according to

$$
S_{2}=\sum_{i}^{N_{C}} x_{i} S_{2, i}
$$

Both the partial- and total nematic order parameters vary conveniently between zero and unity for an isotropic and perfectly ordered nematic phase, respectively.

\section{The Helmholtz energy functional in terms of the Onsager Trial Function}

In this section, the Onsager-Vega-Lago Helmholtz energy functional as derived in Sec. III A (Eqs. (2)-(6)) is evaluated in terms of the OTF (Eq. (18)). We show that for an excluded volume interaction given by Eq. (10), analytical results in terms of the variational parameters of the OTF can be obtained. By using appropriate expansions in these parameters, the Helmholtz energy can be put in compact algebraic form.

Let us first be concerned with the ideal contribution to the Helmholtz energy. Substituting the OTF in Eq. (5), followed by the substitution $u=\cos (\theta)$ and integration by parts leads to the following analytical result:

$$
a_{\text {aniso }}^{\text {id }}=\sum_{i}^{N_{C}} \rho_{i}\left\{\ln \left[\alpha_{i} \operatorname{coth}\left(\alpha_{i}\right)\right]-1+\frac{\arctan \left(\sinh \left(\alpha_{i}\right)\right)}{\sinh \left(\alpha_{i}\right)}\right\} .
$$

For large values of $\alpha_{i}$ (say $\alpha_{i}>800$ ), the hyperbolic terms in this equation become too large to be naively computed on a regular computer; therefore, for $\alpha_{i}>125$, we approximate the above result to within the machine epsilon of a 64-bit double precision (error $<2^{-53}$ ) as

$$
a_{\text {aniso }}^{\mathrm{id}}=\sum_{i}^{N_{C}} \rho_{i}\left\{\ln \left[\alpha_{i}\right]-1\right\} .
$$

Clearly, the introduced error can be neglected. The derivative of the ideal Helmholtz energy contribution with respect to $\alpha_{i}$, which is needed for solving Eq. (19), can now be obtained as

$$
\left(\frac{\partial a^{\mathrm{id}}}{\partial \alpha_{i}}\right)_{T \rho, \alpha_{j \neq i}}=\rho_{i}\left\{\frac{1}{\alpha_{i}}+\frac{\arctan \left[\sinh \left(\alpha_{i}\right)\right] \cosh \left(\alpha_{i}\right)}{\sinh ^{2}\left(\alpha_{i}\right)}\right\} .
$$

For $\alpha_{i}>125$, this is approximated as (error $<2^{-53}$ )

$$
\left(\frac{\partial a^{\mathrm{id}}}{\partial \alpha_{i}}\right)_{T \rho, \alpha_{j \neq i}}=\frac{\rho_{i}}{\alpha_{i}}
$$

Let us now focus on the residual Helmholtz energy contribution (Eq. (6)). To evaluate this term, we need to solve the second virial coefficient (Eqs. (8) and (9)) in terms of the variational parameters of the OTF. Using the excluded volume expression from Eq. (10), the second virial coefficient of any two molecules 1 and 2 of type $i$ and $j$, respectively, can be written as

$$
\begin{aligned}
B_{2, i j}\left[f_{i}\left(\boldsymbol{\omega}_{1}\right), f_{j}\left(\boldsymbol{\omega}_{2}\right)\right]= & \frac{1}{2} V_{\bar{m}_{i j}}\left[C_{1, i j}+C_{2, i j}\langle\sin (\gamma)\rangle_{i j, \omega_{1}, \omega_{2}}\right. \\
& \left.+C_{3, i j}\left\langle\sin ^{2}(\gamma)\right\rangle_{i j, \omega_{1}, \omega_{2}}\right] .
\end{aligned}
$$

The evaluation of the orientational averages of the $\sin (\gamma)$ - and $\sin ^{2}(\gamma)$-kernels of the pair-excluded volume is of central importance for the calculation of the second virial coefficient and thus the residual Helmholtz energy - of the nematic fluid. In terms of the OTF, these averages can be derived as

$$
\begin{aligned}
\langle\sin (\gamma)\rangle_{i j, \omega_{1}, \omega_{2}}= & \frac{1}{2 \sinh \left(\alpha_{i}\right) \sinh \left(\alpha_{j}\right)} \\
& \times \int_{\gamma=0}^{\pi} \cosh \left(\sqrt{\alpha_{i}^{2}+\alpha_{j}^{2}+2 \alpha_{i} \alpha_{j} \cos (\gamma)}\right) \\
& \times \cos (\gamma) \mathrm{d} \gamma,
\end{aligned}
$$

$$
\begin{aligned}
\left\langle\sin ^{2}(\gamma)\right\rangle_{i j, \omega_{1}, \omega_{2}}= & \frac{1}{\sinh \left(\alpha_{i}\right) \sinh \left(\alpha_{j}\right)} \\
& \times \int_{\gamma=0}^{\pi} \cosh \left(\sqrt{\alpha_{i}^{2}+\alpha_{j}^{2}+2 \alpha_{i} \alpha_{j} \cos (\gamma)}\right) \\
& \times \cos (\gamma) \sin (\gamma) \mathrm{d} \gamma
\end{aligned}
$$

For a detailed analysis on the derivation of these two integrals, the reader is referred to the comprehensive work of FrancoMelgar et al. ${ }^{54,63}$ For brevity, this derivation is here omitted. Although, in principle, both of the above integrals can be evaluated numerically, it is much more attractive to use an analytical - albeit approximate - solution instead. The reason for this is twofold. First, both integrals are calculated inside a double iteration-loop for solving for the equilibrium orientational state of the system (Eq. (19)) and phase equilibrium (Eqs. (14) and (15)), respectively. As a consequence, their computation (and that of the required numerical derivatives) is intensive. Second, for systems with very high orientational order, say $\alpha_{i}$ $>800$ (which can happen in the dense nematic phase), these 
terms become too large to be naively computed on a regular computer. We devote the remainder of this section to obtaining approximate solutions of Eqs. (27) and (28).

The first integral (Eq. (27)) was approximated by Onsager in the Appendix of his seminal paper from 1949. ${ }^{9}$ Since from Onsager's analysis it is difficult to subtract for which cases his approximation is justified, we will here go through his derivation in a bit more detail and put some emphasis on the assumptions made. Let us first make the following substitution:

$$
\alpha_{i}^{2}+\alpha_{j}^{2}+2 \alpha_{i} \alpha_{j} \cos (\gamma)=\left(\alpha_{i}+\alpha_{j}-t\right)^{2} .
$$

Using this, we obtain two solutions for the lower and upper boundary of integration, i.e., $t_{l}=0$ or $t_{l}=2\left(\alpha_{i}+\alpha_{j}\right)$ and $t_{u}$ $=2 \alpha_{i}$ or $t_{u}=2 \alpha_{j}$, respectively. Although the choice for a specific solution is arbitrary, it is convenient to choose $t_{l}=0$ and $t_{u}=2 \alpha_{k}$ where $k$ is the index of the component with the lowest degree of orientational order of the pair $i j$. For the purely repulsive molecules considered in this work, this component will always be the less elongated one (see Sec. IV A). It can be verified that by choosing these boundaries, the range of integration is such that the factor $t-\alpha_{i}-\alpha_{j}$ is always smaller than - or equal to - zero; therefore, the hyperbolic terms can be approximated by an exponential as

$$
\begin{aligned}
\frac{\cosh \left(\alpha_{i}+\alpha_{j}-t\right)}{2 \sinh \left(\alpha_{i}\right) \sinh \left(\alpha_{j}\right)} & =\frac{\exp \left(\alpha_{i}+\alpha_{j}-t\right)+\exp \left(t-\alpha_{i}-\alpha_{j}\right)}{4 \sinh \left(\alpha_{i}\right) \sinh \left(\alpha_{j}\right)} \\
& \approx \frac{\exp \left(\alpha_{i}+\alpha_{j}\right)}{4 \sinh \left(\alpha_{i}\right) \sinh \left(\alpha_{j}\right)} \exp (-t) \\
& \approx \exp (-t) .
\end{aligned}
$$

To complete the transformation of variables, we write

$$
\cos (\gamma) \mathrm{d} \gamma=\cos (\gamma) \frac{\mathrm{d} \gamma}{\mathrm{d} t} \mathrm{~d} t=\frac{\mathrm{d} \sin (\gamma)}{\mathrm{d} t} \mathrm{~d} t
$$

which, combined with Eqs. (27) and (30), leads to

$$
\langle\sin (\gamma)\rangle_{i j, \omega_{1}, \omega_{2}}=\int_{0}^{2 \alpha_{k}} \exp (-t) \frac{\mathrm{d} \sin (\gamma)}{\mathrm{d} t} \mathrm{~d} t .
$$

To proceed, we obtain $\sin (\gamma)$ from the trigonometric identity $\sin (\gamma)=\sqrt{1-\cos ^{2}(\gamma)}$, with $\cos (\gamma)$ evaluated from Eq. (29) as $\cos (\gamma)=1-t / \alpha_{i}-t / \alpha_{j}+t^{2} /\left(2 \alpha_{i} \alpha_{j}\right)$. After some rearrangements, we obtain

$$
\sin (\gamma)=\sqrt{2 t\left(\frac{\alpha_{i}+\alpha_{j}}{\alpha_{i} \alpha_{j}}\right)} \sqrt{1-x},
$$

where

$$
\begin{aligned}
x= & \frac{3 t}{2\left(\alpha_{i}+\alpha_{j}\right)}+\frac{t}{2\left(\alpha_{i}+\alpha_{i}^{2} / \alpha_{j}\right)}+\frac{t}{2\left(\alpha_{j}+\alpha_{j}^{2} / \alpha_{i}\right)} \\
& -\frac{t^{2}}{2\left(\alpha_{i} \alpha_{j}+\alpha_{i}^{2}\right)}-\frac{t^{2}}{2\left(\alpha_{i} \alpha_{j}+\alpha_{j}^{2}\right)}+\frac{t^{3}}{8\left(\alpha_{i} \alpha_{j}^{2}+\alpha_{i}^{2} \alpha_{j}\right)}
\end{aligned}
$$

Onsager proceeded by expanding the square root $\sqrt{1-x}$ around $x=0$ (as $1-x / 2-x^{2} / 8-x^{3} / 16-\ldots$ ) and truncating after second order in $x$. Before we do this, it is instructive to look at the behavior of $x$ as a function of the relevant

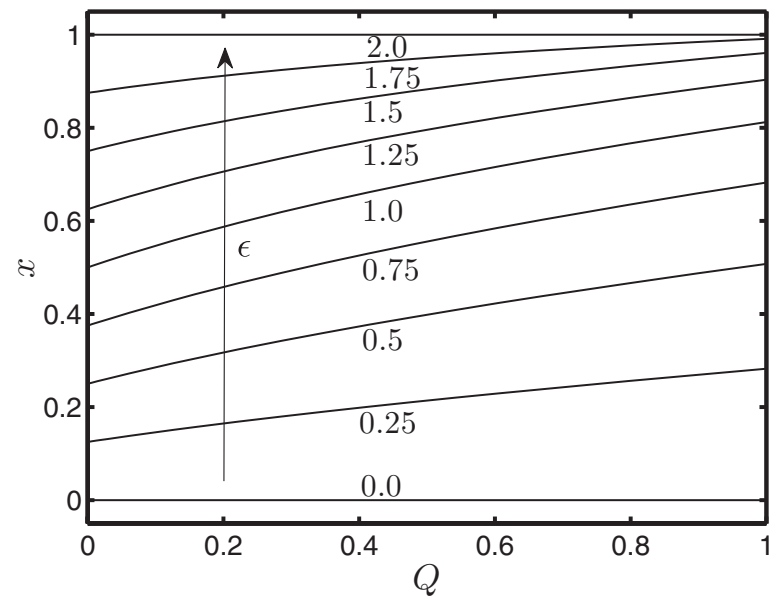

FIG. 1. The $x$-parameter from Eq. (34) versus the ratio of orientational or$\operatorname{der} Q=\alpha_{1} / \alpha_{2}$ (where $\alpha_{2} \geq \alpha_{1}$ ) for various values of $t=\epsilon \cdot \alpha_{1}$, with $\epsilon=0.0,0.25,0.5, \ldots, 2.0$. The expansion $\sqrt{1-x}=1-x / 2-x^{2} / 8-$ $x^{3} / 16-\ldots$ converges only when $x<1$.

parameters, and gain some insight into the accuracy of this expansion. As imposed by the limits of integration of Eq. (32), $t=\epsilon \cdot \alpha_{k}$, where $0 \leq \epsilon \leq 2$. Accordingly, one can rewrite the above equation for $x$ in terms of $\epsilon$ and a newly introduced variable $Q=\alpha_{i} / \alpha_{j}$, where $i=k$ is chosen as the component with the lowest orientational order (i.e., $0 \leq Q \leq 1$ ). Different diagrams of $x$ versus $Q$ for $\epsilon=0,0.25,0.5, \ldots, 2.0$ (Fig. 1) show that the expansion is justified as long as $\epsilon$ is small. For $\epsilon$ close to 2, on the other hand, the value of $x$ tends to unity, leading to a decreased accuracy of the expansion. For $x=1$, the expansion is not justified. Consequently, only close to the upper boundary of Eq. (32) the use of the expansion constitutes a non-negligible degree of approximation to the integrand. Whether this decrease in accuracy will affect the calculated result from Eq. (32) depends on the value of $\alpha_{k}$. For any reasonable value of $\alpha_{k}$ (say, 5 or larger) the exponential will be the dominant factor in the integrand; thereby scaling any errors introduced by the expansion of the square root to approximately zero. For smaller values of $\alpha_{k}$, which occur for very bidisperse mixtures (see Sec. IV A), some error in the computed results can be expected. Performing the expansion, taking the derivative to $t$ and truncating after third order in $\alpha$, leads to the following result:

$$
\begin{aligned}
\frac{\mathrm{d} \sin (\gamma)}{\mathrm{d} t}= & \sqrt{\frac{\alpha_{i}+\alpha_{j}}{2 \alpha_{i} \alpha_{j}}}\left\{\frac{1}{\sqrt{t}}-\left[\frac{9 \sqrt{t}}{4\left(\alpha_{i}+\alpha_{j}\right)}+\frac{3 \sqrt{t}}{4\left(\alpha_{i}+\alpha_{i}^{2} / \alpha_{j}\right)}\right.\right. \\
& +\frac{3 \sqrt{t}}{4\left(\alpha_{j}+\alpha_{j}^{2} / \alpha_{i}\right)}-\frac{5 t^{3 / 2}}{4\left(\alpha_{i} \alpha_{j}+\alpha_{i}^{2}\right)} \\
& \left.-\frac{5 t^{3 / 2}}{4\left(\alpha_{i} \alpha_{j}+\alpha_{j}^{2}\right)}+\ldots\right]-\frac{1}{32}\left[\frac{45 t^{3 / 2}}{\left(\alpha_{i}+\alpha_{j}\right)^{2}}\right. \\
& +\frac{5 t^{3 / 2}}{\left(\alpha_{i}+\alpha_{i}^{2} / \alpha_{j}\right)^{2}}+\frac{5 t^{3 / 2}}{\left(\alpha_{j}+\alpha_{j}^{2} / \alpha_{i}\right)^{2}}
\end{aligned}
$$




$$
\begin{aligned}
& +\frac{10 t^{3 / 2}}{\left(\alpha_{i}+\alpha_{i}^{2} / \alpha_{j}\right)\left(\alpha_{j}+\alpha_{j}^{2} / \alpha_{i}\right)} \\
& +\frac{30 t^{3 / 2}}{\left(\alpha_{i}+\alpha_{j}\right)\left(\alpha_{i}+\alpha_{i}^{2} / \alpha_{j}\right)} \\
& \left.\left.+\frac{30 t^{3 / 2}}{\left(\alpha_{i}+\alpha_{j}\right)\left(\alpha_{j}+\alpha_{j}^{2} / \alpha_{i}\right)}+\ldots\right]\right\} .
\end{aligned}
$$

Substitution in Eq. (32) leads to a series of integrals of the form $K_{n}\left(\alpha_{i}, \alpha_{j}\right) \int_{0}^{2 \alpha_{k}} \exp (-t) t^{n / 2} \mathrm{~d} t$ where $n=-1,1$ or 3 , respectively, and $K_{n}\left(\alpha_{i}, \alpha_{j}\right)$ is a $t$-independent factor to be determined from Eq. (35). As discussed, for a sufficiently large value of the variational parameter $\alpha_{k}$, the exponential forces the value of the integrand to zero close to the upper boundary of integration, allowing for an extension of the range of integration to $t=\left[\begin{array}{ll}0 & \infty\end{array}\right]$. This extension is particularly useful since now a transformation $t=y^{2}$ can be used to reduce these integrals to the following standard Gaussian types: $\int_{0}^{\infty} \exp \left(-y^{2}\right) \mathrm{d} y=\sqrt{\pi / 4}, \int_{0}^{\infty} \exp \left(-y^{2}\right) y^{2} \mathrm{~d} y=\sqrt{\pi / 16}$ and $\int_{0}^{\infty} \exp \left(-y^{2}\right) y^{4} \mathrm{~d} y=(3 / 8) \sqrt{\pi}$. Now, we obtain Onsager's approximation for the orientational average of the $\sin (\gamma)$ kernel of the pair-excluded volume after rearranging

$$
\begin{aligned}
& \langle\sin (\gamma)\rangle_{i j, \omega_{1}, \omega_{2}} \\
& =\sqrt{\frac{\pi}{2}\left(\frac{\alpha_{i}+\alpha_{j}}{\alpha_{i} \alpha_{j}}\right)}\left\{1-\frac{3}{8}\left[\frac{1}{\alpha_{i}+\alpha_{j}}+\frac{1}{\alpha_{i}}+\frac{1}{\alpha_{j}}+\ldots\right]\right. \\
& \left.\quad+\frac{15}{128}\left[\frac{8}{\alpha_{i} \alpha_{j}}-\left(\frac{1}{\alpha_{i}+\alpha_{j}}+\frac{1}{\alpha_{i}}+\frac{1}{\alpha_{j}}\right)^{2}+\ldots\right]\right\} .
\end{aligned}
$$

For completeness, the percentage difference of the above analytical result compared to its exact numerical counterpart (Eq. (27)) is shown for a grid in $\alpha_{i}$ and $\alpha_{j}$ in Fig. 2. For the larger part of parameter space, the approximation is remarkably accurate; showing a negligible relative difference $\left(<10^{-3}\right)$ for both $\alpha$-parameters larger than 5. As expected, the approximation breaks down if one of the $\alpha$-parameters ap-

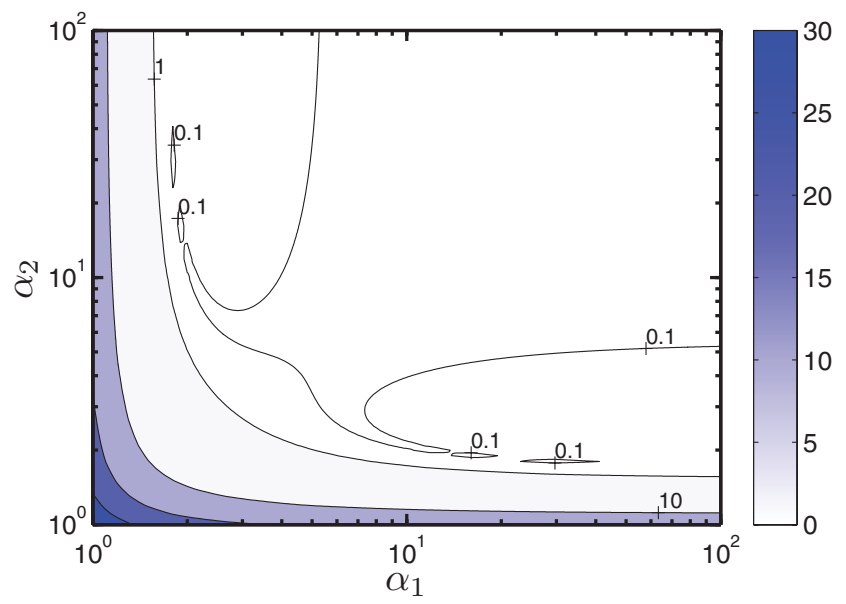

FIG. 2. The percentage deviation of the analytical approximation for the orientational average of the $\sin (\gamma)$-kernel of the pair-excluded volume from Eq. (36) compared to the full numerical solution from Eq. (27). proaches unity; limiting its application to systems of not too extreme bidispersity. For all systems considered in this work, however, we will find that the bidispersity is moderate enough to use Eq. (36) as an accurate approximation of Eq. (27).

To the best of our knowledge, no analytical solution of the second integral (Eq. (28)) has thus far been presented in literature. Most probably, the reason is that for typical rigid, hard-core nematogens studied, the orientation dependence of the pair-excluded volume can be captured by a first order term in $\sin (\gamma)$. For the partially flexible molecules studied in this work, however, the incorporation of a second order term in $\sin (\gamma)$ is required. ${ }^{50}$ In many respects, the method followed to evaluate Eq. (28) is the same as that laid out by FrancoMelgar et al. ${ }^{54,63}$ for the pure-component fluid. We show that, as for the pure-component case, exact analytical results can be obtained.

To proceed, let us start with the transformation of variables $a=\alpha_{i}^{2}+\alpha_{j}^{2}, b=2 \alpha_{i} \alpha_{j}$, and $u=\sqrt{a+b \cos (\gamma)}$, which leads to

$$
\begin{aligned}
& \mathrm{d} \gamma=-\frac{2 u \mathrm{~d} u}{b \sin (\gamma)}, \\
& \cos (\gamma)=\frac{u^{2}-a}{b} .
\end{aligned}
$$

Substitution in Eq. (28) results in

$$
\begin{aligned}
\left\langle\sin ^{2}(\gamma)\right\rangle_{i j, \omega_{1}, \omega_{2}} & \\
= & \frac{2}{\sinh \left(\alpha_{i}\right) \sinh \left(\alpha_{j}\right)}\left\{\frac{a}{b^{2}} \int_{\alpha_{i}+\alpha_{j}}^{\alpha_{i}-\alpha_{j}} \cosh (u) u \mathrm{~d} u\right. \\
& \left.\quad-\frac{1}{b^{2}} \int_{\alpha_{i}+\alpha_{j}}^{\alpha_{i}-\alpha_{j}} \cosh (u) u^{3} \mathrm{~d} u\right\}
\end{aligned}
$$

which can be solved straightforwardly by successive integration by parts. After rearranging, we obtain the following exact analytical result

$$
\begin{aligned}
\left\langle\sin ^{2}(\gamma)\right\rangle_{i j, \omega_{1}, \omega_{2}} & \frac{1}{2 \sinh \left(\alpha_{i}\right) \sinh \left(\alpha_{j}\right)} \\
\quad & \quad\left\{\sinh \left(\alpha_{i}-\alpha_{j}\right)\left(\frac{-2}{\alpha_{i}}+\frac{2}{\alpha_{j}}-\frac{6}{\alpha_{i} \alpha_{j}^{2}}+\frac{6}{\alpha_{i}^{2} \alpha_{j}}\right)\right. \\
& -\cosh \left(\alpha_{i}-\alpha_{j}\right)\left(\frac{6}{\alpha_{i} \alpha_{j}}-\frac{6}{\alpha_{i}^{2} \alpha_{j}^{2}}-\frac{2}{\alpha_{i}^{2}}-\frac{2}{\alpha_{j}^{2}}\right) \\
& +\sinh \left(\alpha_{i}+\alpha_{j}\right)\left(\frac{2}{\alpha_{i}}+\frac{2}{\alpha_{j}}+\frac{6}{\alpha_{i} \alpha_{j}^{2}}+\frac{6}{\alpha_{i}^{2} \alpha_{j}}\right) \\
& \left.-\cosh \left(\alpha_{i}+\alpha_{j}\right)\left(\frac{2}{\alpha_{i}^{2}}+\frac{2}{\alpha_{j}^{2}}+\frac{6}{\alpha_{i} \alpha_{j}}+\frac{6}{\alpha_{i}^{2} \alpha_{j}^{2}}\right)\right\} .
\end{aligned}
$$

For $\alpha_{i}=\alpha_{j}$ this reduces to the pure-component result obtained by Franco-Melgar et al. ${ }^{54,63}$ Also, using the hyperbolic properties $\cosh (-x)=\cosh (x)$ and $\sinh (-x)=-\sinh (x)$, it can be verified that the above result is symmetric in $i$ and $j$. As mentioned earlier, the computation of the hyperbolic 
terms can become problematic for systems of very high orientational order. It is therefore useful to simplify the above result a little further. Using the hyperbolic properties $\sinh (x$ $+y)=\sinh (x) \cosh (y)+\cosh (x) \sinh (y)$ and $\cosh (x+y)$ $=\cosh (x) \cosh (y)+\sinh (x) \sinh (y)$, we can write

$$
\begin{aligned}
\frac{\sinh \left(\alpha_{i}-\alpha_{j}\right)}{\sinh \left(\alpha_{i}\right) \sinh \left(\alpha_{j}\right)}=\operatorname{coth}\left(\alpha_{j}\right)-\operatorname{coth}\left(\alpha_{i}\right) \\
\frac{\cosh \left(\alpha_{i}-\alpha_{j}\right)}{\sinh \left(\alpha_{i}\right) \sinh \left(\alpha_{j}\right)}=\operatorname{coth}\left(\alpha_{i}\right) \operatorname{coth}\left(\alpha_{j}\right)-1 \\
\frac{\sinh \left(\alpha_{i}+\alpha_{j}\right)}{\sinh \left(\alpha_{i}\right) \sinh \left(\alpha_{j}\right)}=\operatorname{coth}\left(\alpha_{j}\right)+\operatorname{coth}\left(\alpha_{i}\right) \\
\frac{\cosh \left(\alpha_{i}+\alpha_{j}\right)}{\sinh \left(\alpha_{i}\right) \sinh \left(\alpha_{j}\right)}=\operatorname{coth}\left(\alpha_{i}\right) \operatorname{coth}\left(\alpha_{j}\right)+1
\end{aligned}
$$

For typical nematic mixtures, the values of the $\alpha$-parameters are larger than 3 ; therefore, to a very good approximation (error $\sim 10^{-3}$ ) the $\operatorname{coth}(.$.$) -terms can be reduced to unity, and$ Eq. (40) can be simplified further to the following algebraic equation:

$$
\begin{aligned}
\left\langle\sin ^{2}(\gamma)\right\rangle_{i j, \omega_{1}, \omega_{2}}= & \frac{2}{\alpha_{i}}+\frac{2}{\alpha_{j}}-\frac{2}{\alpha_{i}^{2}}-\frac{2}{\alpha_{j}^{2}}-\frac{6}{\alpha_{i} \alpha_{j}} \\
& +\frac{6}{\alpha_{i} \alpha_{j}^{2}}+\frac{6}{\alpha_{i}^{2} \alpha_{j}}-\frac{6}{\alpha_{i}^{2} \alpha_{j}^{2}}
\end{aligned}
$$

For all systems considered in the present work, Eq. (42) is used to approximate the orientational average of the $\sin ^{2}(\gamma)$ kernel from Eq. (28). As shown in Fig. 3, the approximation is excellent, showing negligible error for a large and relevant part of parameter space. Similar to the approximation of the $\sin (\gamma)$-kernel (Fig. 2), the approximation of the $\sin ^{2}(\gamma)$ kernel breaks down if the $\alpha$-parameter of one (or both) of the components approaches unity.

To solve Eq. (19) for the equilibrium orientational state of the system, we also need the derivatives of Eqs. (36) and (42) to $\alpha_{i}$. For configurations between molecules of different

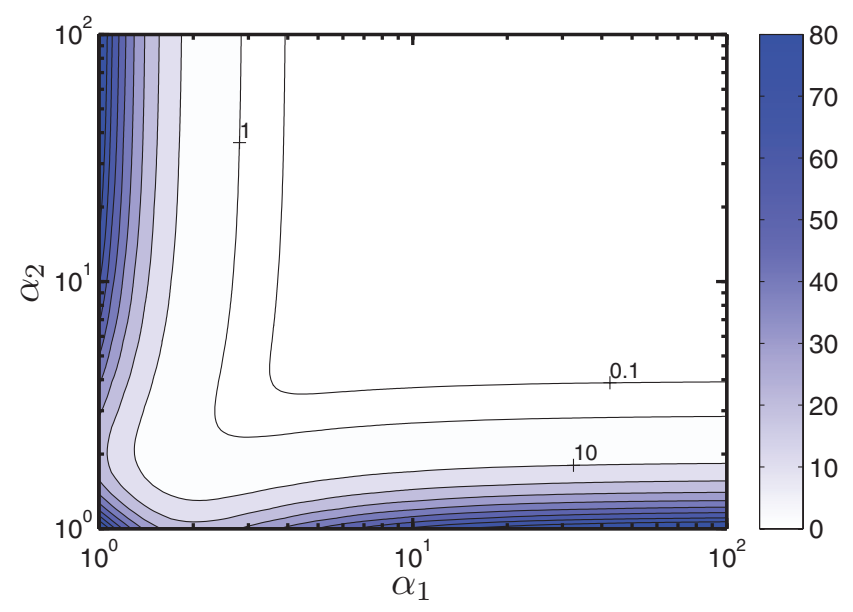

FIG. 3. The percentage deviation of the analytical approximation for the orientational average of the $\sin ^{2}(\gamma)$-kernel of the pair-excluded volume from Eq. (40) compared to the full numerical solution from Eq. (28). type, i.e., $i \neq j$, these can be derived as, respectively,

$$
\begin{aligned}
& \left(\frac{\partial\langle\sin (\gamma)\rangle_{i j, \omega_{1}, \omega_{2}}}{\partial \alpha_{i}}\right)_{\alpha_{j}} \\
& =-\sqrt{\frac{\pi \alpha_{j}}{8 \alpha_{i}^{4}+8 \alpha_{j} \alpha_{i}^{3}}}\left\{1-\frac{3}{8}\left[\frac{1}{\alpha_{i}+\alpha_{j}}+\frac{1}{\alpha_{i}}+\frac{1}{\alpha_{j}}\right]\right. \\
& \left.\quad+\frac{15}{128}\left[\frac{8}{\alpha_{i} \alpha_{j}}-\left(\frac{1}{\alpha_{i}+\alpha_{j}}+\frac{1}{\alpha_{i}}+\frac{1}{\alpha_{j}}\right)^{2}\right]\right\} \\
& \quad+\sqrt{\frac{\pi}{2}\left(\frac{\alpha_{i}+\alpha_{j}}{\alpha_{i} \alpha_{j}}\right)}\left\{\frac{3}{8}\left[\frac{1}{\left(\alpha_{i}+\alpha_{j}\right)^{2}}+\frac{1}{\alpha_{i}^{2}}\right]\right. \\
& \left.\quad+\frac{15\left[\frac{-8}{128}+2\left(\frac{1}{\alpha_{i}^{2} \alpha_{j}}+\alpha_{j}\right.\right.}{\alpha_{i}}+\frac{1}{\alpha_{i}}\right) \\
& \left.\left.\quad \times\left(\frac{1}{\left(\alpha_{i}+\alpha_{j}\right)^{2}}+\frac{1}{\alpha_{i}^{2}}\right)\right]\right\}
\end{aligned}
$$

$$
\begin{aligned}
\left(\frac{\partial\left\langle\sin ^{2}(\gamma)\right\rangle_{i j, \omega_{1}, \omega_{2}}}{\partial \alpha_{i}}\right)_{\alpha_{j}}= & -\frac{2}{\alpha_{i}^{2}}-\frac{4}{\alpha_{i}^{3}}+\frac{6}{\alpha_{i}^{2} \alpha_{j}}-\frac{6}{\alpha_{i}^{2} \alpha_{j}^{2}} \\
& -\frac{12}{\alpha_{i}^{3} \alpha_{j}}+\frac{12}{\alpha_{i}^{3} \alpha_{j}^{2}}
\end{aligned}
$$

For configurations between molecules of the same type, i.e., $i=j$, we obtain

$$
\begin{gathered}
\frac{\mathrm{d}\langle\sin (\gamma)\rangle_{i i, \omega_{1}, \omega_{2}}}{\mathrm{~d} \alpha_{i}}=\sqrt{\pi}\left\{\frac{45}{32 \alpha_{i}^{5 / 2}}-\frac{1}{2 \alpha_{i}^{3 / 2}}-\frac{525}{1024 \alpha_{i}^{7 / 2}}\right\} \\
\frac{\mathrm{d}\left\langle\sin ^{2}(\gamma)\right\rangle_{i i, \omega_{1}, \omega_{2}}}{\mathrm{~d} \alpha_{i}}=-\frac{4}{\alpha_{i}^{2}}+\frac{20}{\alpha_{i}^{3}}-\frac{36}{\alpha_{i}^{4}}+\frac{24}{\alpha_{i}^{5}}
\end{gathered}
$$

In summary, we have derived analytical results for the orientation-dependent parts of the ideal- (Eqs. (22) and (23)) and residual (Eqs. (36) and (42)) Helmholtz energy contribution in terms of the variational parameters of the OTF. Also the derivatives of these contributions to the variational parameters have been obtained (Eqs. (24) and (25) and (43)(46)). In Sec. IV, these approximate results are used in the Onsager-Vega-Lago (OVL) theory that was laid out in Sec. III A. An analytical EoS for the isotropic and nematic phase of tangent hard-sphere chain fluid mixtures is thereby obtained. This EoS will be referred to as truncated OVL theory. When the exact (numerical) solutions of the orientational parts of the residual Helmholtz energy are used instead (Eqs. (27) and (40)), it will simply be referred to as OVL theory. It is shown later that both theories result in essentially identical results for the phase diagram of binary tangent hard-sphere chain fluid mixtures. 


\section{RESULTS AND DISCUSSION}

\section{A. The effect of length bidispersity on the I-N and N-N phase behavior}

In this section, we analyze the effect of length bidispersity on the isotropic-nematic and nematic-nematic phase behavior of binary mixtures of linear tangent hard-sphere chain fluids. The effect of length bidispersity on the degree of orientational order of the system is also studied. Since the analysis is purely theoretical, it is of value to test the theory to results from molecular simulations first. To the best of our knowledge, the only suitable simulation data available is that of Escobedo and de Pablo, ${ }^{24}$ who used an expanded Gibbs-ensemble Monte Carlo (MC) method to simulate the I-N pressure-composition envelope of a mixture of linear 8mers and linear 16-mers. In Fig. 4, we compare their results to those obtained from the truncated OVL theory developed in this work. Results from the OVL theory are also included for comparison. The theoretical results are in excellent agreement to simulation data. The results obtained from both theories are virtually indistinguishable, showing the accuracy of the truncated form of the OVL theory. Both theory and simulation show (equally) strong fractionation of the two components between the two phases, with the more anisotropic 16-mer fluid preferentially partitioned in the nematic phase.

We view the favorable comparison between theory and simulation as a strong indicator that the developed theory accurately captures the phase behavior of bidisperse mixtures, which encourages a further analysis of phase equilibria. We define a bidispersity ratio $q=m_{1} / m_{2}$, and vary $q$ systematically by changing the length of component 2 (where $m_{2}$ $>m_{1}$ ). The length of component 1 is fixed at $m_{1}=8$. Using this procedure we were able to locate two types of phase diagrams, shown in Fig. 5 for four different systems of decreasing bidispersity ratio. For a bidispersity ratio close to unity,

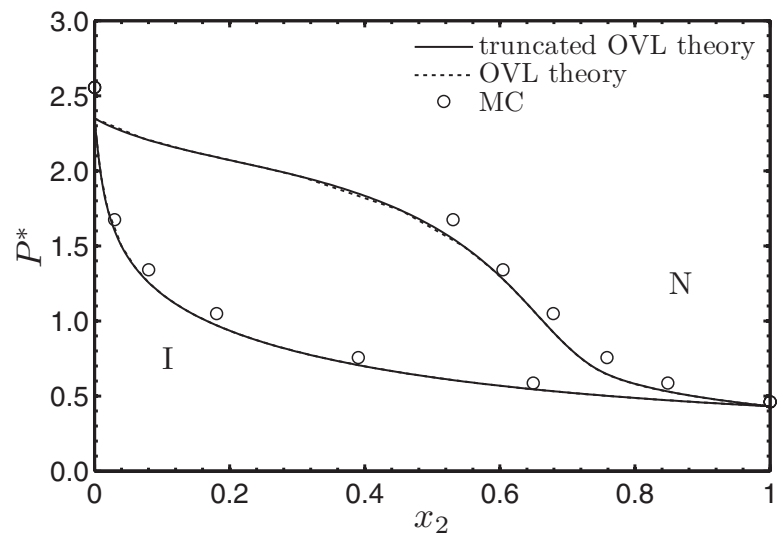

FIG. 4. A comparison between the dimensionless pressure $P^{*}=\beta P V_{m=8}$ of a binary mixture of linear 8-mers and linear 16-mers as obtained from the truncated OVL theory (solid line), the full numerical solution of the OVL theory (dotted line), and MC simulations ${ }^{24}$ (symbols). Here, $x_{2}$ is the mole fraction of 16-mers, $V_{m=8}=(\pi / 6) m \sigma^{3}$ is the molecular volume of the linear 8 -mer molecules. The results obtained from the full numerical solution of the OVL theory are almost indistinguishable from those obtained from the truncated variant. For the sake of comparison to the MC simulations, the focus of this figure is on the isotropic-nematic (I-N) coexistence; therefore, the nematic-nematic coexistence exhibited at higher pressure is not shown. we find I-N coexistence at low pressure and a N-N demixed region with a lower critical point at higher pressure (Figs. 5(a) and 5(b)). With decreasing bidispersity ratio, the N-N critical point is shifted to lower pressure until, for $m_{2}=19$, the N$\mathrm{N}$ region starts to overlap with the I-N region, resulting in a triphase I-N-N equilibrium at $P^{*}=P V_{m=8} / k T=2.234$, $x_{2, \mathrm{I}}=0.000631, x_{2, \mathrm{~N}^{-}}=0.111$ and $x_{2, \mathrm{~N}^{+}}=0.428$ (Fig. 5(c)). Here, we have introduced the notation $\mathrm{N}^{-}$and $\mathrm{N}^{+}$for the coexisting nematic phases lean and rich in the longer component, respectively. If the bidispersity ratio is decreased further, an $\mathrm{I} \rightarrow \mathrm{N} \rightarrow \mathrm{I} \rightarrow \mathrm{N}$ re-entrant phenomenon ${ }^{15-17,20,23}$ is observed upon increasing the pressure in some parts of the phase diagram (Fig. 5(d)). For completeness, the phase diagrams from Fig. 5 are shown in a $\eta-x$ representation in Fig. 6.

It is important to note that we have tried to probe parameter space more rigorously by using different molecule types for the reference component 1 , using more extreme bidispersity ratio's (down to 0.0001 ) and considering non-integer values of the chain length. Nonetheless, we did not find any other types of phase diagrams than those shown in Fig. 5, suggesting that (considering only I and $\mathrm{N}$ phases) this is the complete picture for binary mixtures of linear tangent hard-sphere chain fluids of equally-sized segments. In a study of Varga et al. ${ }^{20}$ it was found that systems of hard rods of equal length but different diameter can also exhibit regions of N-N coexistence bounded by an upper critical point and regions of I-I coexistence. Given the similarity to the molecular model studied in this work, it seems reasonable to suggest these types of phase behavior will also be observed for binary mixtures of tangent hard-sphere chain fluids of non-equally-sized segments.

Since the results for the N-N region have not been tested by comparing to $\mathrm{MC}$ simulations, let us elaborate on the accuracy of the theoretical results. As discussed in Sec. III C, the approximations related to truncating the OVL theory become more reliable for large values of the variational parameters (see Figs. 2 and 3). Therefore, any errors introduced by using these approximations within the framework of the OVL theory can be neglected in the highly ordered $\mathrm{N}-\mathrm{N}$ region. The OVL framework itself (see Sec. III A), however, is expected to become less accurate at this part of the phase diagram. The OVL description of nematic phases relies on approximating higher virial coefficients; therefore, provided that an accurate description of the isotropic phase is available, the N-N equilibrium will be inevitably less accurately described than the I-N equilibrium. Also, compared to the I-N region, the N-N region is located at relatively higher pressure/density; therefore, any errors introduced by the approximation of higher virial coefficients will become more pronounced. To what extent these errors influence the theoretical results is unclear at this point. For an assessment on this, a systematic MC study on the N-N behavior would be desirable; however, we realize that due to the high densities involved, conveying such a study is non-trivial.

In contrast to the I-N transition, which was explained by Onsager as the result of a competition between orientational and configurational/excluded volume entropy, the nature of the N-N demixing transition has been a point of debate for some time. What can be subtracted from the available 
(a)
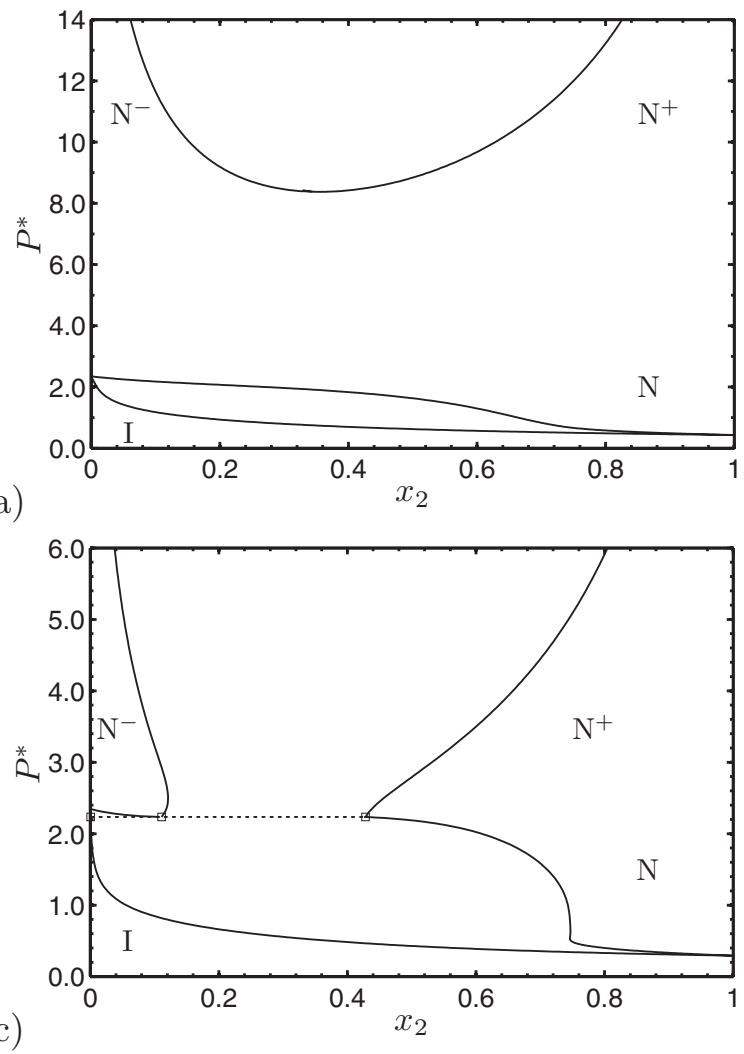

(b)
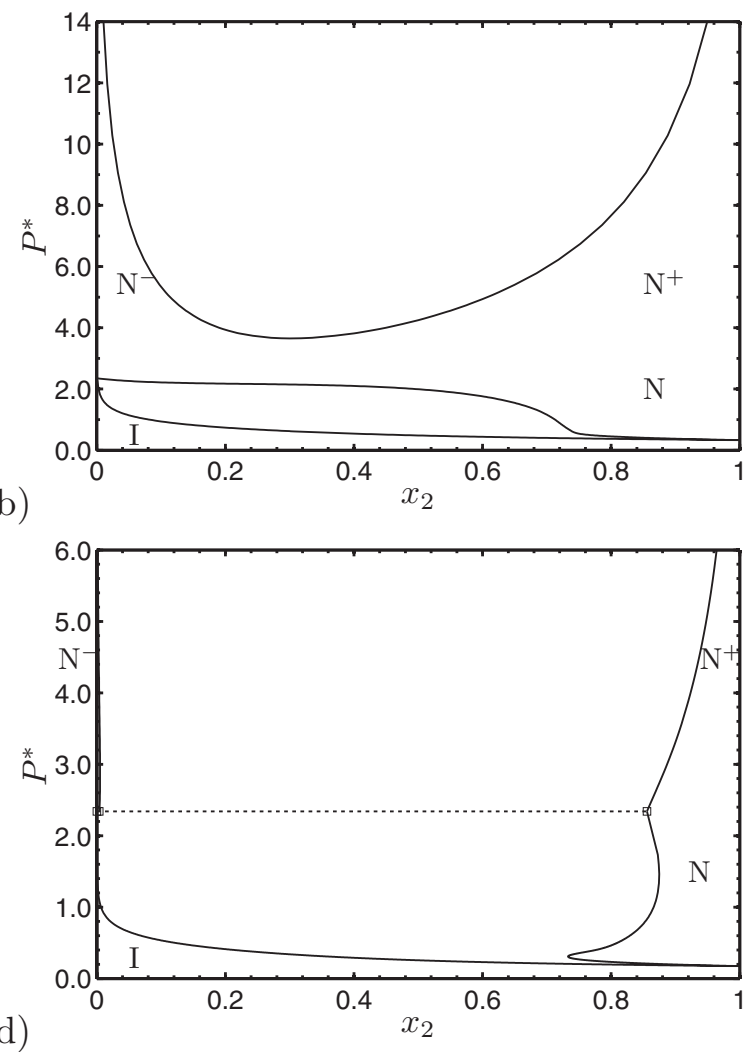

FIG. 5. The dimensionless pressure $P^{*}=\beta P V_{m=8}$ of a binary mixture of linear 8-mers and (a) linear 16-mers, (b) linear 18-mers, (c) linear 19-mers, and (d) linear 24-mers, as obtained from the truncated OVL theory. Here, $x_{2}$ is the mole fraction of the long component in the mixture, $V_{m=8}=(\pi / 6) m \sigma^{3}$ is the molecular volume of the linear 8-mer molecules. With increasing pressure, coexistence between an isotropic (I) and nematic (N) phase- and two nematic phases lean $\left(\mathrm{N}^{-}\right)$and rich $\left(\mathrm{N}^{+}\right)$in the long component is observed, respectively. The $\mathrm{I}-\mathrm{N}^{-}-\mathrm{N}^{+}$threephase equilibrium is denoted by the dotted line.

literature on this point is that, depending on the molecular architecture of the components in the mixture, both orientational- and configurational/excluded volume entropy can give rise to a N-N demixing transition. ${ }^{16,20,27}$ To obtain insight into the driving force behind the N-N demixing transition of the tangent hard-sphere chain fluids studied here, we analyze the behavior of the total Gibbs energy and its individual (entropic) contributions with varying composition of the system. The total dimensionless Gibbs energy $\tilde{g}=\beta G / N$ is obtained from the Helmholtz energy using the thermodynamic relation

$$
\tilde{g}=\frac{\beta A}{N}+Z .
$$

Here, $Z=\beta P / \rho$ is the compressibility factor of the system. One should note that in calculating the total Helmholtz energy, the density-independent factor $\Lambda_{i}^{3} / \Omega$ from Eq. (4) has not been considered since it is of no importance for the location of the phase equilibrium. Accordingly, the individual contributions to $\tilde{g}$ arising from, respectively, translational-, mixing-, orientational-, and configurational/excluded volume entropy, are obtained from Eqs. (4) and (5) as

$$
\begin{gathered}
\tilde{g}_{\text {trans }}=\ln \rho, \\
\tilde{g}_{\text {mix }}=\sum_{i} x_{i} \ln x_{i}, \\
\tilde{g}_{\text {orient }}=\sum_{i} x_{i}\left(\ln \alpha_{i}-1\right),
\end{gathered}
$$

$$
\tilde{g}_{\text {conf }}=\frac{\beta A^{\text {res }}}{N}+Z^{\text {res }} .
$$

The total Gibbs energy from Eq. (47) is then retained from the sum $\tilde{g}=\tilde{g}_{\text {trans }}+\tilde{g}_{\text {mix }}+\tilde{g}_{\text {orient }}+\tilde{g}_{\text {conf. }}$. Since for purely repulsive molecules the N-N demixing transition does not occur in pure component systems, it is sufficient for our analysis to consider excess properties. Hence, we subtract the properties of the ideal mixture (i.e., a mole-fraction-weighted sum of pure-component thermodynamic properties) according to

$$
\begin{gathered}
\tilde{g}^{\mathrm{ex}}=\tilde{g}-\sum_{i} x_{i} \tilde{g}_{i}^{0}, \\
\tilde{g}_{\text {trans }}^{\mathrm{ex}}=\tilde{g}_{\text {trans }}-\sum_{i} x_{i} \tilde{g}_{i, \text { trans }}^{0} \\
\ldots \text { etc. }
\end{gathered}
$$

This procedure allows for a meaningful graphical representation of the demixing transition in a $\tilde{g}-x$ diagram, because the dominant linear contributions from Eqs. (48)-(51) are subtracted. Subtracting the linear contributions does not alter the location of the phase transition as obtained from a common tangent-line construction. ${ }^{64}$ In Fig. 7, we show a plot of the excess Gibbs energy and its individual contributions versus the composition of a binary mixture of linear 8-mers and 18-mers at a pressure $P^{*}=\beta P V_{m=8}=10.3494$. The results clearly show that both the translational and mixing entropy favor the (single phase) mixed state. The contributions 
(a)
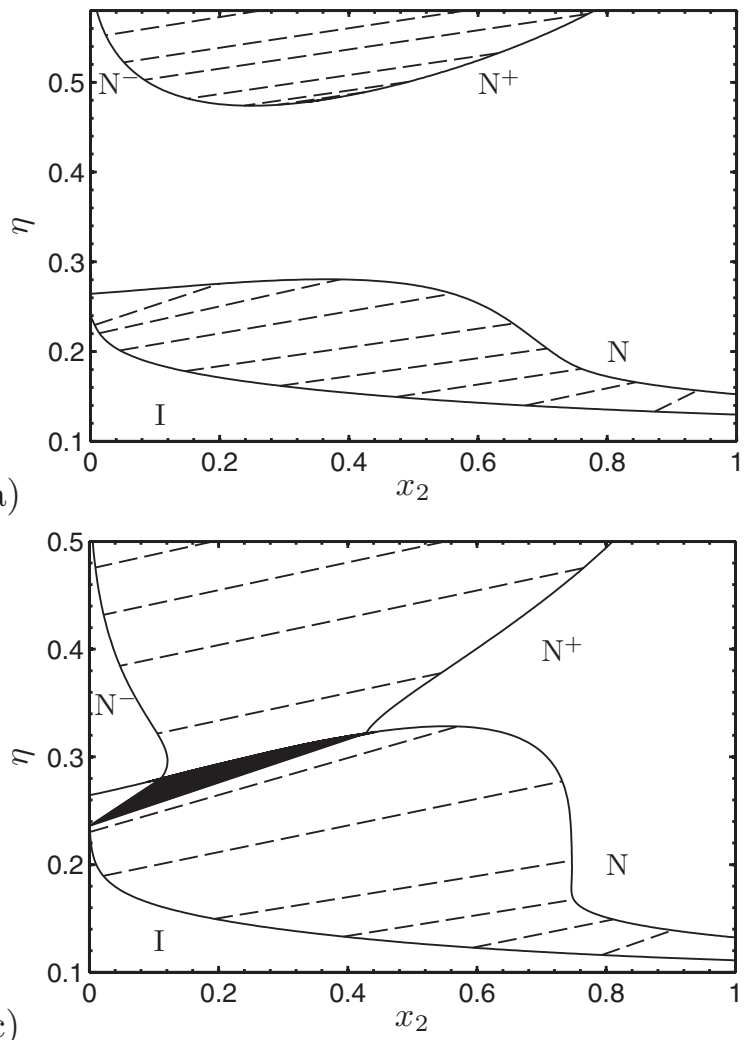

(b)

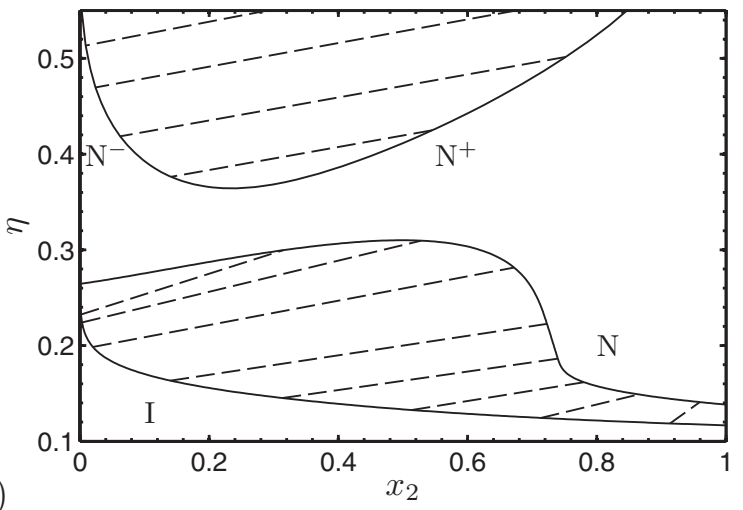

(d)

FIG. 6. The packing fraction $\eta=\rho(\pi / 6) \sum_{i} x_{i} m_{i} \sigma^{3}$ in the coexisting isotropic and nematic phase of a binary mixture of linear 8-mers and (a) linear 16-mers, (b) linear 18-mers, (c) linear 19-mers, and (d) linear 24-mers, as obtained from the truncated OVL theory. Here, $x_{2}$ is the mole fraction of the long component in the mixture, $V_{m=8}=(\pi / 6) m \sigma^{3}$ is the molecular volume of the linear 8-mer molecules. The $\mathrm{I}-\mathrm{N}^{-}-\mathrm{N}^{+}$threephase equilibrium is denoted by the black shaded region.

arising from orientational- and configurational/excluded volume entropy favor a demixing transition to two nematic phases. In this respect, these results are comparable to those obtained by Varga et al. ${ }^{20}$ who found that, for systems of thin and thick hard rods, both orientational- and configurational entropy favor N-N demixing. The results are different than those obtained by Wensink et al. ${ }^{27}$ who found that, for systems of thin and thick hard platelets, the N-N demixing transition is driven by configurational entropy only.

Let us now shift focus to the orientational order parameters. As shown in some previous studies, ${ }^{14,15,23}$ the degree of orientational order of the components in a nematic binary mixture is very sensitive to composition. In Fig. 8 we show this behavior is also observed for the tangent hard-sphere chain model studied in this work. Three binary mixtures are considered. For all three mixtures, the first component is a linear 8-mer. The second component is varied between a linear 10-mer (Fig. 8(a)), 14-mer (Fig. 8(b)) or 18-mer (Fig. 8(c)), respectively. It can be observed that over the whole composition range, the orientational order of the long component is significantly larger than that of the short component. The reason is that at a certain density of the system, the shorter molecules have more freedom to rotate their axes away from the nematic director than the longer molecules. For systems of purely repulsive molecules, this behavior is general. It can also be observed that the orientational order parameter has a maximum with composition. With decreasing bidispersity ratio $q=m_{1} / m_{2}$, the maximum becomes more pronounced and takes place at larger values of the mole fraction of the long component. These observations can be explained from a competition between two effects: (1) a decrease of the I$\mathrm{N}$ coexistence pressure with increasing mole fraction of the long component (resulting in a decrease in density - and thus orientational order - of the nematic phase), and (2) induction

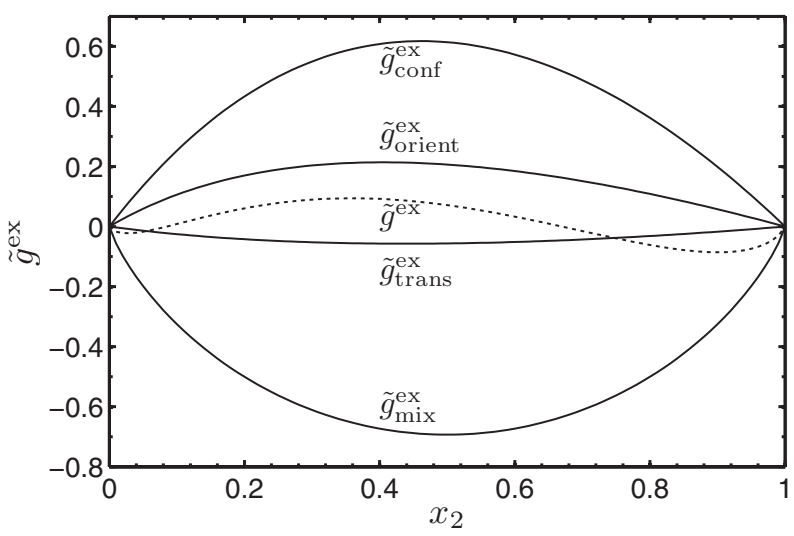

FIG. 7. The total excess Gibbs energy (dotted line) for a nematic phase of linear 8-mers and linear 18-mers at a pressure $P^{*}=\beta P V_{m=8}=10.3494$. Here, $x_{2}$ is the mole fraction of linear 18 -mers, $V_{m=8}=(\pi / 6) m \sigma^{3}$ is the molecular volume of the linear 8-mer molecules. Results are obtained from the truncated OVL theory. The solid lines correspond to the individual contributions to the Gibbs energy, arising from, translational- $\left(\tilde{g}_{\text {trans }}^{\mathrm{ex}}\right)$, orientational$\left(\tilde{g}_{\text {orient }}^{\text {ex }}\right)$, mixing- $\left(\tilde{g}_{\text {mix }}^{\text {ex }}\right)$, and configurational/excluded volume entropy $\left(\tilde{g}_{\text {conf }}^{\text {ex }}\right)$, respectively. 
(a)

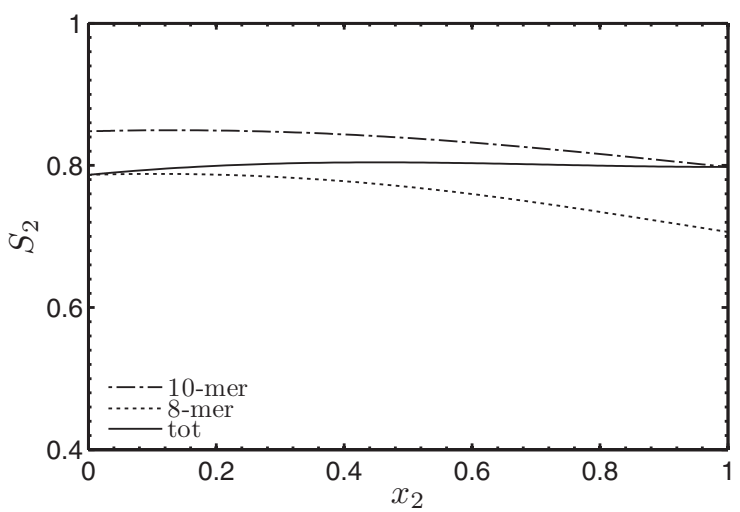

(b)

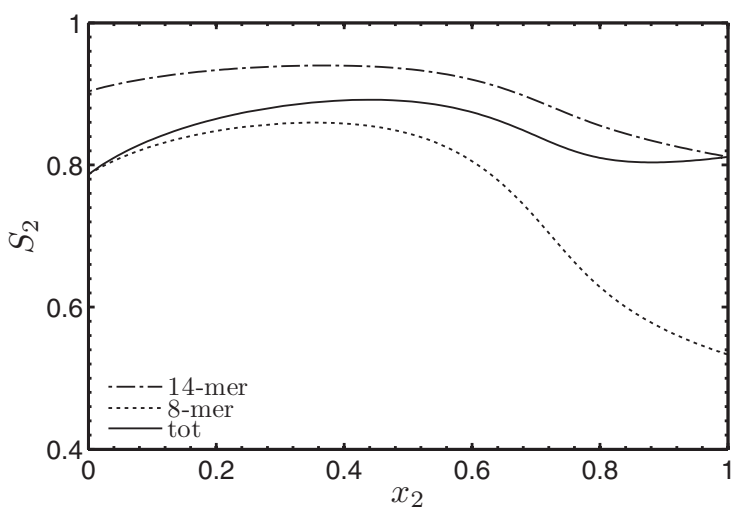

(c)

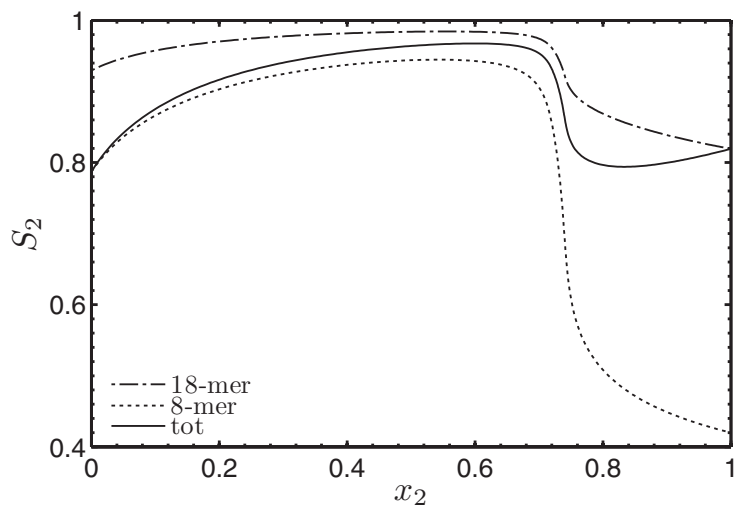

FIG. 8. The I-N coexistence values of the total- and partial nematic order parameters $S_{2}$ (Eq. (21)) and $S_{2, i}$ (Eq. (20)), respectively, of a binary mixture of linear 8-mers and (a) linear 10-mers, (b) linear 14-mers, and (c) linear 18-mers, as obtained from the truncated OVL theory. Here, $x_{2}$ is the mole fraction of the long component in the nematic mixture.

of orientational order by adding a long component to a short component. In principle, when a long component is added to a nematic phase rich in a short component, the I-N coexistence pressure is much higher than it would be for the pure long component. Consequently, the degree of orientational order of the long component in the mixture is much higher than the typical coexistence value (i.e., $S_{2} \sim 0.8$ ) found for the pure component system. As a result, the degree of orientational order of the short component is also increased (which we hereby refer to as "induction"). Note that this second effect is enhanced by the fractionation of the longer and shorter molecules between the phases.

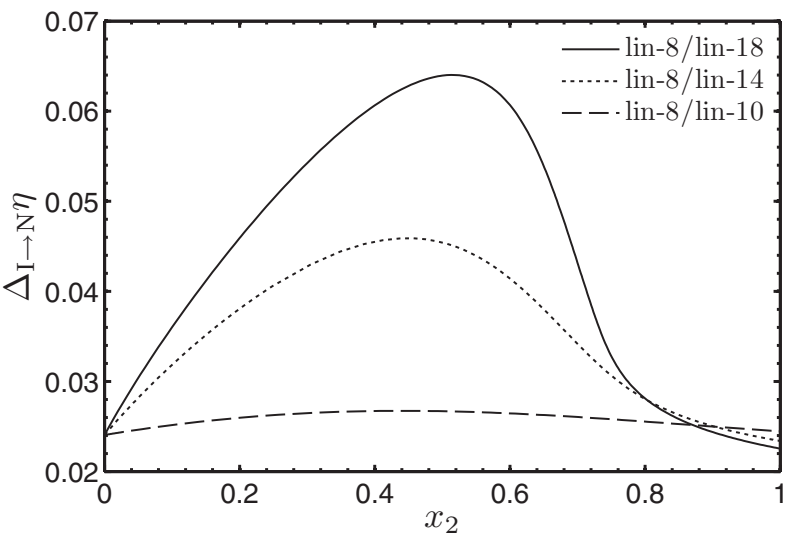

FIG. 9. The difference of the packing fraction $\eta=\rho(\pi / 6) \sum_{i} x_{i} m_{i} \sigma^{3}$ in the coexisting isotropic and nematic phase for the same binary mixtures as those shown in Fig. 8. Here, $x_{2}$ is the mole fraction of the long component in the nematic phase mixture. Results were obtained from the truncated OVL theory.

The maximum in the orientational order has an interesting implication. As shown in Fig. 9, it results in a maximum difference in density between the coexisting isotropic and nematic phase. As shown in a previous MC simulation study, ${ }^{65}$ this density difference is a key factor determining a step-wise decrease in solubility of small gases across the I-N phase transition. In the light of applying LC solvents for gas absorption processes, ${ }^{66,67}$ this observation is interesting, since it means that, as for many applications of LCs, the properties of a LC solvent can be optimized by using a LC mixture of specific composition. Below, in Sec. IV C we further elaborate on this point, but for now, we investigate the effect of partial molecular flexibility on the phase diagram.

\section{B. The effect of partial molecular flexibility on the I-N and $\mathrm{N}-\mathrm{N}$ phase behavior}

Let us define the binary mixture of linear 8-mers and linear 19-mers (Fig. 5(c)) as a reference. To study the effect of partial molecular flexibility we gradually make the 19-mer more flexible by means of the rod-coil model laid out in Sec. II; considering a number of 1 or 2 segments in the flexible tail, respectively. The rod-coils considered are thus the 19-18 and 19-17. The phase diagrams are presented in Fig. 10. As for linear chain fluids, the truncated form of the OVL theory proves to be very accurate, leading to essentially identical results as obtained from the full numerical solution of the theory. It can be observed that, when compared to linear systems (Fig. 5), an increase in flexibility leads to the same topological changes in the phase diagram as a decrease in chain length of the more elongated component, namely a reduced fractionation of components between the I and $\mathrm{N}$ phase, a shift of the N-N critical point to higher pressure, and disappearance of the triphase I-N-N equilibrium. The reason for this similarity is that an increase in flexibility of the second (rod-coil) component reduces the length of its rigid block, and thereby "effectively" increases the bidispersity ratio $q=m_{1} / m_{2}$. 
(a)

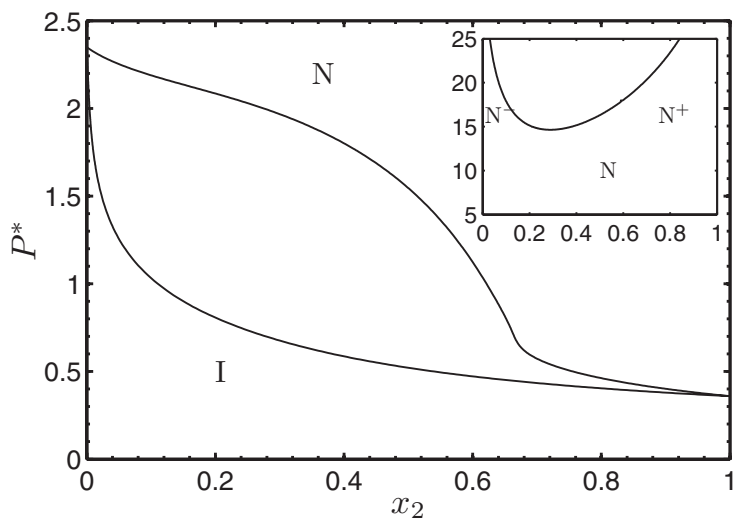

(b)

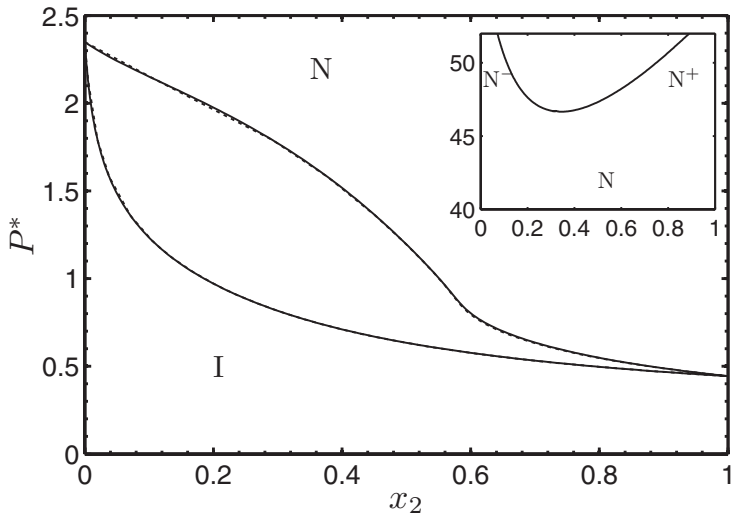

FIG. 10. The dimensionless pressure $P^{*}=\beta P V_{m=8}$ of a binary mixture of linear 8-mers and (a) 19-18 rod-coils, and (b) 19-17 rod-coils, as obtained from the truncated OVL theory (solid lines). Here, $x_{2}$ is the mole fraction of the rod-coils, $V_{m=8}=(\pi / 6) m \sigma^{3}$ is the molecular volume of the linear 8-mer molecules. For (b), the results from the numerical solution of the OVL theory are included as well (dotted lines); the results of both theories are essentially indistinguishable. With increasing pressure, coexistence between an isotropic (I) and nematic $(\mathrm{N})$ phase and two nematic phases lean $\left(\mathrm{N}^{-}\right)$and rich $\left(\mathrm{N}^{+}\right)$in the long component is observed, respectively. The insets show the $\mathrm{N}^{-}-\mathrm{N}^{+}$demixed region which, due to the partial flexibility of the second component in the mixture, is shifted to very high pressure.

In spite of the topological similarity of the phase diagrams of linear- and rod-coil fluids, there is a pronounced difference in the location of the N-N critical points. For example, the lower $\mathrm{N}-\mathrm{N}$ critical point of a binary fluid of linear 18-mers and linear 8-mers (Fig. 5(b)) is located at much lower pressure than that of a binary fluid of 19-18 rod-coils and linear 8-mers (Fig. 10(a)), despite the fact that the number of beads in the rigid block is equal for the 18-mer and 19-18 rod-coil. Due to the presence of the flexible tail, the pair-excluded volume of the 19-18 rod-coil fluid is less anisotropic than that of the linear 18-mer fluid. ${ }^{50}$ Hence, for the binary fluid of linear 8-mers and 19-18 rod-coils, there is less configurational entropy that can be gained by demixing into two different nematic phases. This is clearly illustrated by comparing the $\tilde{g}-x$ diagram from Fig. 11 (linear 8-mer/19-18 rod-coil mixture) to that from Fig. 7 (linear 8-mer/linear 18-mer mixture). In conclusion, when adding a flexible tail to one of the linear chain molecules of a binary mixture, the driving force for $\mathrm{N}-\mathrm{N}$ demixing is

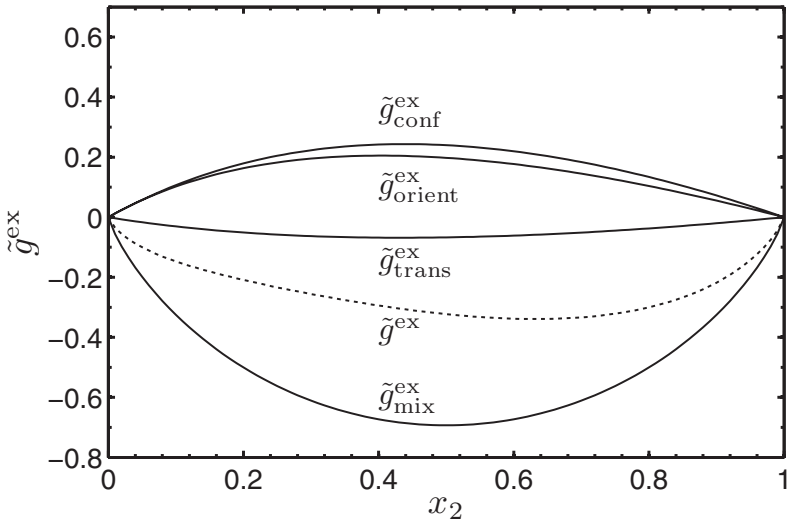

FIG. 11. The total excess Gibbs energy (dotted line) versus composition of a nematic phase of linear 8-mers and 19-18 rod-coils at a pressure $P^{*}=\beta P V_{m=8}=10.3494$. Here, $x_{2}$ is the mole fraction of the 19-18 rod-coils, $V_{m=8}=(\pi / 6) m \sigma^{3}$ is the molecular volume of the linear 8 -mer molecules. Results are obtained from the truncated OVL theory. The solid lines correspond to the individual contributions to the Gibbs energy, arising from, translational- $\left(\tilde{g}_{\text {trans }}^{\text {ex }}\right)$, orientational- $\left(\tilde{g}_{\text {orient }}^{\text {ex }}\right)$, mixing- $\left(\tilde{g}_{\text {mix }}^{\text {ex }}\right)$, and configurational/excluded volume entropy ( $\tilde{g}_{\text {conf }}^{\text {ex }}$ ), respectively. Compared to Fig. 7 , where a similar diagram is shown for a binary fluid of linear 8-mers and linear 18-mers, the configurational contribution to the Gibbs energy is lower, resulting in a smaller driving force for $\mathrm{N}-\mathrm{N}$ demixing. As a result, the $\mathrm{N}-\mathrm{N}$ demixing transition is shifted to higher pressure than considered in this figure.

decreased, leading to a shift of the N-N transition to higher density/pressure.

\section{Solubility of hard-sphere solutes in linear and partially flexible tangent hard-sphere chain solvents}

As a measure for the solubility of hard-sphere (hs) solutes in tangent hard-sphere chain solvents, we introduce the Henry's law constant $H_{k}$, formally defined by ${ }^{68}$

$$
H_{k}=\lim _{x_{k} \rightarrow 0}\left(\frac{f_{k}^{L}}{x_{k}}\right),
$$

where $k$ is the hs component, and $x_{k}$ and $f_{k}^{L}(V, T, \underline{x})$ are the mole fraction and fugacity of this component in the liquid (i.e., isotropic or nematic) phase, respectively. In terms of the fugacity coefficient $\phi_{k}^{L}=f_{k}^{L} /\left(x_{k} P\right)=\exp \left[\beta \mu_{k}^{\text {res }}\right]$, where $P$ is the pressure exerted by the system and $\mu_{k}^{\text {res }}(V, T, \underline{x})$ is the residual chemical potential of component $k$, the above definition can be rewritten as

$$
H_{k}=\lim _{x_{k} \rightarrow 0}\left(\phi_{k}^{L} P\right)=\lim _{x_{k} \rightarrow 0}\left(\exp \left[\beta \mu_{k}^{\text {res }}\right] P\right),
$$

where the residual chemical potential is obtained from

$$
\beta \mu_{k}^{\mathrm{res}}=\left(\frac{\partial a^{\mathrm{res}}\left[f_{\mathrm{eq}}(\boldsymbol{\omega})\right]}{\partial \rho_{k}}\right)_{\rho_{j \neq k} T} .
$$

For convenience, we define a modified Henry's law constant $H_{k}^{\prime}$, according to

$$
H_{k}^{\prime}=\frac{H_{k}}{P}=\lim _{x_{k} \rightarrow 0}\left(\exp \left[\beta \mu_{k}^{\mathrm{res}}\right]\right) .
$$

The motivation for using this modified Henry's law constant is merely to allow for a better graphical comparison between the Henry's law constants of hs solutes in different tangent hard-sphere chain solvents. 


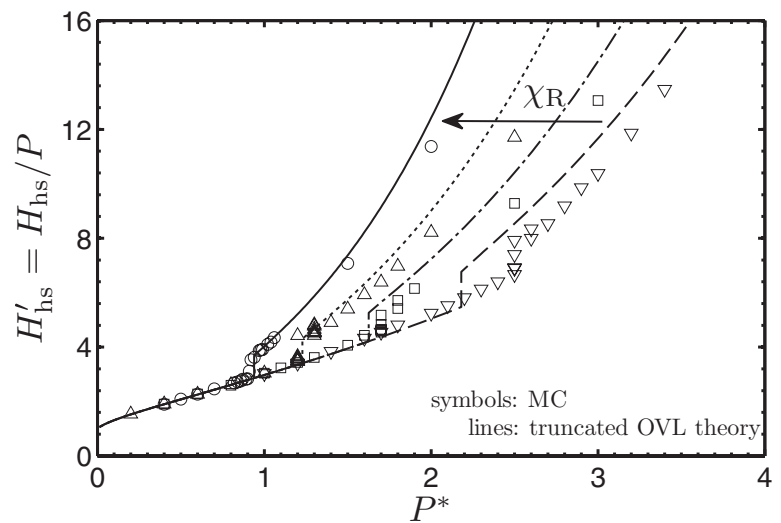

FIG. 12. Modified Henry's law constant of a hs solute in several hard-chain solvents as a function of the dimensionless pressure $P^{*}=\beta P V_{m}$, where $V_{m}=(\pi / 6) m \sigma^{3}$ is the molecular volume of the solvent molecules of chain length $m$. The hard-chain solvents considered are, (from left to right) in order of decreasing $\chi_{\mathrm{R}}$, a linear 15-mer, a 15-14 rod-coil, a 15-13 rod-coil, and a 15-12 rod-coil. Comparison of theoretical results obtained from the truncated OVL theory (lines) and MC simulation data from Ref. 65 (symbols).

In Fig. 12, we compare the calculated modified Henry's law constant of a hs solute in a linear 15-mer-, a 15-14 rodcoil-, a 15-13 rod-coil-, and a 15-12 rod-coil fluid to MC simulations. ${ }^{65}$ For the linear 15 -mer solvent, excellent agreement between simulation and theory is obtained. For the partially flexible LC solvents, agreement is satisfactory; deteriorating somewhat with flexibility. For all systems shown, a step-wise increase of the modified Henry's law constant is observed across the I-N transition; corresponding to a step-wise decrease in solubility of the hs solute.

As shown in a previous MC simulation study, ${ }^{65}$ this solubility difference is caused primarily by the density difference between the coexisting I and $\mathrm{N}$ phase. The orientational ordering was shown to have no - or at most a negligible effect. In the simulations, a bias function was used to prescribe orientational order independent of density. The advantage of such a method is, that the effect of ordering on the Henry's law constant can be singled out for a defined density of the system. The theory can equally be applied with a prescribed value of orientational order. In Fig. 13 we emphasize this, by considering the modified Henry's law constant of a hs solute in a linear 15 -mer solvent with a varying orientational order parameter $S_{2}$ at constant density of the system. Agreement between theory and simulations is excellent. As can be observed, when singled out from density, the orientational ordering has no significant effect on the modified Henry's law constant - and thus the solubility - of the hs solute.

Given this result, and the observation that for binary LC mixtures the density difference between the coexisting I and $\mathrm{N}$ phase shows a maximum with composition (Fig. 9), we conclude that the solubility difference of small solutes across the I-N phase transition can be maximized by choosing a binary LC mixture of specific composition as the solvent. To illustrate this, we calculated how the Henry's law constant of a hs solute changes when a binary LC solvent of linear 8-mers and linear 18-mers changes from the isotropic phase to the nematic phase. The results are displayed for varying composition of the LC solvent in Fig. 14. The results for the den-

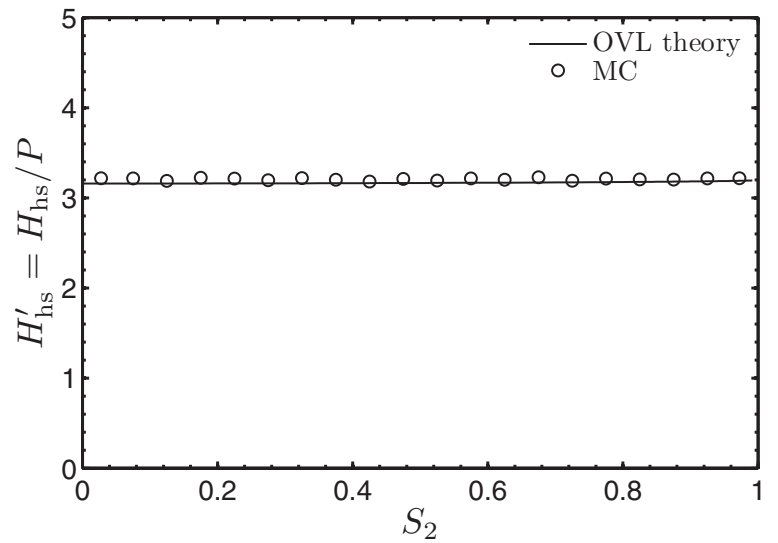

FIG. 13. Modified Henry's law constant of a hs solute in a linear 15-mer solvent with varying orientational order parameter of the system. The density of the system is fixed at $\eta=0.147$, which is in between the isotropic and nematic coexistence density of the pure solvent. Comparison of theoretical results obtained from the OVL theory (line) and MC simulation data from Ref. 65 (symbols).

sity difference across the phase transition are included as well. Both quantities are related, and - like the density difference - the solubility difference displays a maximum with composition. The composition dependence is remarkably strong, resulting in approximately a factor 3 increase of the solubility difference (compared to the pure solvents) at a mole fraction of the linear 18-mer of $x \approx 0.5$.

Whether these results can be extrapolated to mixtures of real LCs depends on several aspects. First, there is the issue of anisotropic attractive intermolecular interactions, such as those arising from dipolar forces. In some studies (see, for example, Refs. 69 and 70), it was proposed that such interactions can result in the formation of dimer complexes, wherein the rigid cores of two LCs are aligned. Such a model was used, for example, to explain the occurrence of induced smectic- and re-entrant nematic phase behavior. Moreover, in two recent experimental studies, ${ }^{71,72}$ this proposed dimerization mechanism was used to explain an observed minimum in the

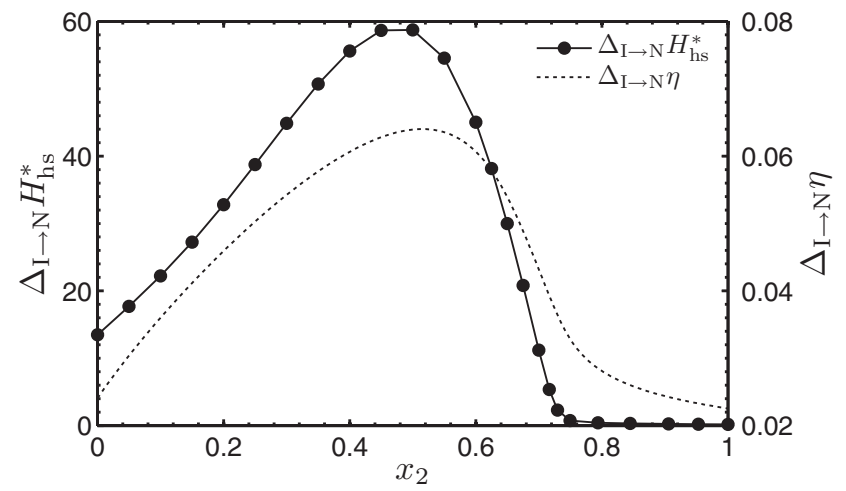

FIG. 14. Left vertical axis: The difference of the isotropic- and nematic coexistence value of the dimensionless Henry's law constant $H^{*}=\beta H V_{m=8}$ of a hard sphere solute in a binary LC solvent of linear 8-mers and linear 18mers. Here, $V_{m=8}=(\pi / 6) m \sigma^{3}$ is the molecular volume of the linear 8 -mer molecules. Right vertical axis: The difference of the isotropic- and nematic coexistence value of the packing fraction of the binary LC solvent. Horizontal axis: The mole fraction of linear 18-mers in the nematic phase. 
nematic order parameter for a certain composition of a binary mixture of dipolar LCs. Clearly, the formation of these complexes should be avoided if one wants to maximize the difference in solubility of small gases across the I-N transition. For such purposes, a maximum in the order parameter is required. For further study, it would be interesting to use molecular simulations to investigate (1) the formation of these dimer complexes, and (2) to what extent they influence the orientational order and solubility of small gases in the mixture. To best of our knowledge, no such study is available in literature.

Second, an important aspect to be considered is the stability of the nematic phase compared to other mesophases. Experiments have shown that in binary mixtures of LCs with a chemically similar core but different length of the (semi-)flexible tail, the smectic phase can become stabilized at the cost of the nematic phase if the length ratio of the tails is increased. ${ }^{6,7,73}$ Since these results could well be an artifact of increased flexibility of the molecules, it would be interesting to test by experiments/molecular simulations if this stabilization of the smectic phase also occurs when only the length ratio of the rigid cores is increased. The LCs used in such a study should be of nematic type. To best of our knowledge, no such study is available in literature. With regard to applying LC solvents as absorption liquids, the formation of smectic phases at the cost of a nematic phase is undesirable, since it could limit the possibility of maximizing the solubility difference across the I-N phase transition.

\section{CONCLUSION}

In summary, we have developed an analytical EoS to describe the isotropic- and nematic phase behavior of linearand partially flexible tangent hard-sphere chain fluids and their mixtures. The EoS is based on a Vega-Lago rescaled Onsager theory in the Onsager Trial Function approximation, and can be considered as an extension of our previous work on pure component systems. ${ }^{33}$ Analytical results for both the Helmholtz energy and compressibility factor were obtained due to the use of an analytical approximation for the pair-excluded volume. ${ }^{50}$ The orientational averages of the $\sin (\gamma)$ - and $\sin ^{2}(\gamma)$-kernel of the pair-excluded volume were approximated analytically using Onsager's truncated asymptotic expansion ${ }^{9}$ and a novel approximation derived in this work, respectively.

The EoS was tested by comparing against molecular simulation data for the I-N coexistence of a binary mixture of linear 8-mers and linear 16-mers. It was also compared to simulation data for the Henry's law constants of a hs solute in different linear and partially flexible tangent hard-sphere chain solvents. Overall agreement of the theory to simulation data is excellent. In particular, the fractionation of the long and short component between the I and $\mathrm{N}$ phase is predicted very accurately. For the Henry's law constants, we found that the accuracy of the theoretical results deteriorates somewhat with the flexibility of the hard-chain solvent - a result not that surprising given the results obtained previously for pure component systems. ${ }^{33}$

From a theoretical point of view, the EoS developed in this work was used to study several phenomena. First, we studied the effect of length bidispersity on the I-N and N$\mathrm{N}$ phase behavior of binary mixtures of linear $m$-mers. For a bidispersity ratio $q=m_{1} / m_{2}$ (where $m_{2} \geq m_{1}$ ) close to unity, we found an I-N coexistence region at lower pressure and a N-N demixed region bounded by a lower critical point at higher pressure. With decreasing bidispersity ratio (i.e., less-like molecules), the N-N critical point is shifted to lower pressure until the N-N and I-N region start to overlap, resulting in a triphase I-N-N equilibrium. If the bidispersity ratio is decreased even further, a re-entrant $\mathrm{I} \rightarrow \mathrm{N} \rightarrow \mathrm{I} \rightarrow \mathrm{N}$ phenomenon can be observed at some parts of the phase diagram. It was shown that the coexistence value of the nematic order parameter of a binary mixture displays a maximum with composition. As a result, the density difference between the coexisting I- and $\mathrm{N}$ phase also shows a maximum with composition. With decreasing bidispersity ratio, the maximum becomes more pronounced.

Second, the effect of partial molecular flexibility on the I-N and N-N phase behavior was studied. Compared to linear chains, no topological difference in the phase diagram was found. However, it was shown that due to a less anisotropic pair-excluded volume, the gain in configurational entropy upon $\mathrm{N}-\mathrm{N}$ demixing was lower than for comparable linear chain fluids, resulting in a smaller driving force for the N-N transition. As a result the N-N demixing transition was shifted to higher density/pressure.

Finally, the solubility of hard-sphere solutes in the isotropic and nematic phase of linear and partially flexible tangent hard-sphere chain solvents was studied. In accordance with the results of a recent MC simulation study, ${ }^{65}$ it was shown that the transition from an isotropic to a nematic phase leads to a step-wise decrease in the solubility of the hardsphere solute. The primary reason for this is a step-wise increase in the density of the system at the phase transition; the orientational ordering alone was shown to have negligible effect. The maximum of the I-N density difference with composition that was found for bidisperse mixtures of linear chain fluids, was utilized to maximize the solubility difference of hard-sphere solutes across the I-N phase transition. These results show that the solubility difference of small gases between a coexisting I- and $\mathrm{N}$ phase can be maximized by using a LC mixture of specific composition as the solvent. In the light of applying LCs as solvents for absorption processes, ${ }^{66,67}$ these results suggest that the direction of research should be directed towards mixtures of LCs.

\section{ACKNOWLEDGMENTS}

The authors would like to thank Dick Bedeaux for helpful discussions on the derivation of the algebraic form of the Helmholtz energy presented in this work. This research is supported by the Stichting voor Technische Wetenschappen (Dutch Technology Foundation, STW), applied science division of the Nederlandse organisatie voor Wetenschappelijk Onderzoek (Netherlands Organization for Scientific Research, NWO) and the Technology Program of the Ministry of Economic Affairs. In addition, this work was sponsored by the Stichting Nationale Computerfaciliteiten (National 
Computing Facilities Foundation, NCF) for the use of supercomputing facilities, with financial support from NWO-EW.

\section{APPENDIX A: GENERALIZATION OF THE OVL THEORY TO MIXTURES}

Starting from the pressure equation for mixtures of nonrigid chain molecules ${ }^{74}$ one can follow the same derivation as presented in Ref. 33 for pure-component systems, and use the decoupling approximation to derive the following virial for the compressibility factor of a nematic fluid mixture

$$
Z=1+\sum_{i} \sum_{j} x_{i} x_{j} g_{i j}\left(1^{+}\right) B_{2, i j}[f(\omega)] \rho .
$$

Here, $g_{i j}\left(1^{+}\right)$is the radial distribution function (rdf) of two molecules of type $i$ and $j$ at contact. We now introduce an averaged rdf $g_{\text {av }}\left(1^{+}\right)$, according to

$$
\begin{aligned}
Z & =1+g_{\text {av }}\left(1^{+}\right) \sum_{i} \sum_{j} x_{i} x_{j} B_{2, i j}[f(\boldsymbol{\omega})] \rho, \\
& =1+g_{\text {av }}\left(1^{+}\right) \bar{B}_{2}[f(\boldsymbol{\omega})] \rho .
\end{aligned}
$$

Assuming $g_{\text {av }}\left(1^{+}\right)$of the nematic fluid can be approximated by that of the isotropic fluid at the same density, one can write

$$
g_{\mathrm{av}}\left(1^{+}\right) \approx g_{\mathrm{av}, \text { iso }}\left(1^{+}\right)=\left(\frac{Z_{\text {iso }}-1}{\rho}\right) \frac{1}{\bar{B}_{2, \text { iso }}}
$$

which, when substituted in Eq. (A2), leads to a generalized OVL theory for nematic fluid mixtures, according to

$$
Z=1+Z_{\text {iso }}^{\text {res }} \frac{\bar{B}_{2}[f(\boldsymbol{\omega})]}{\bar{B}_{2, \text { iso }}} .
$$

It is important to note that, compared to pure component systems, the above derived result for mixtures is more approximate. The reason is that, due to the averaging of the rdf over all components in the mixture, part of the many-fluid behavior is lost. The accurate comparison of theory and molecular simulation results obtained in this work (see Secs. IV A and IV C), however, suggests that the larger part of this behavior is retained by considering all individual pair-interactions of the fluid mixture in the second virial coefficient.

\section{APPENDIX B: THE LH-rC EOS FOR THE ISOTROPIC FLUID}

In a previous work, ${ }^{53}$ we extended the $\mathrm{LH} \mathrm{EoS}^{56,57}$ to describe linear- and partially flexible tangent hard-sphere chain fluids and their mixtures. The extension was based on a refitting of one (a) of the three model constants $(a, b, c)$ to the 2 nd virial coefficient of linear and partially flexible chain fluids (as obtained analytically from Eq. (10)). The other two model constants $(b, c)$ were adjusted to molecular simulation data for the pressure of linear tangent hard-sphere chain fluids in the isotropic phase. Only pure components were incorporated in the fitting. For the $b$ and $c$ parameters, a linear dependence on molecular rigidity (via the rigidity parameter $\chi_{\mathrm{R}}$ as defined in Sec. II) was assumed.
In terms of the compressibility factor, the LH-rc EoS can be written as

$$
Z_{\text {iso }}=\frac{1+a \eta+b \eta^{2}-c \eta^{3}}{(1-\eta)^{3}},
$$

where the $a, b$, and $c$ parameters are defined as

$$
\begin{aligned}
a & =\sum_{i}^{N_{C}} x_{i} m_{i}\left[1+\frac{m_{i}-1}{m_{i}} a_{2}+\frac{m_{i}-1}{m_{i}} \frac{m_{i}-2}{m_{i}} a_{3, i}\right], \\
b & =\sum_{i}^{N_{C}} x_{i} m_{i}\left[1+\frac{m_{i}-1}{m_{i}} b_{2}+\frac{m_{i}-1}{m_{i}} \frac{m_{i}-2}{m_{i}} b_{3, i}\right], \\
c & =\sum_{i}^{N_{C}} x_{i} m_{i}\left[1+\frac{m_{i}-1}{m_{i}} c_{2}+\frac{m_{i}-1}{m_{i}} \frac{m_{i}-2}{m_{i}} c_{3, i}\right],
\end{aligned}
$$

with $a_{2}=0.45696 ; b_{2}=2.10386 ; c_{2}=1.75503$ and

$$
\begin{gathered}
a_{3, i}=p(1)+p(2) \chi_{\mathrm{R}, i}+p(3) \chi_{\mathrm{R}, i}^{2}+p(4) \chi_{\mathrm{R}, i}^{3}, \\
b_{3, i}=3.49695-3.81467 \chi_{\mathrm{R}, i}, \\
c_{3, i}=4.83207-1.35191 \chi_{\mathrm{R}, i} .
\end{gathered}
$$

Here, $\boldsymbol{p}=(-0.74745,0.29915,1.08727,-0.70898)$.

\section{APPENDIX C: COEFFICIENTS FOR CALCULATING THE PAIR-EXCLUDED VOLUME}

In our previous work, ${ }^{50}$ we developed an accurate analytical approximation for the orientation-dependent pairexcluded volume of rod-coil molecules. The functional form of this approximation is given by Eq. (10) of the main text. The coefficients of Eq. (10) are given by

$$
\begin{array}{r}
C_{1}\left(\bar{m}, \bar{\chi}_{\mathrm{R}}\right)=\frac{11 \bar{m}-3}{\bar{m}}\left[1-D\left(\bar{m}, \bar{\chi}_{R}\right)\right] \\
+\frac{\left(m_{1}-1\right)\left(m_{2}-1\right)}{\bar{m}} \sum_{k=1}^{3} a_{k}\left(1-\bar{\chi}_{\mathrm{R}}\right)^{k}, \\
C_{2}\left(\bar{m}, \bar{\chi}_{\mathrm{R}}\right)=3.5339 \frac{\left(m_{1}-1\right)\left(m_{2}-1\right)}{\bar{m}} \bar{\chi}_{\mathrm{R}}^{2} \\
C_{3}\left(\bar{m}, \bar{\chi}_{\mathrm{R}}\right)=\frac{\left(m_{1}-1\right)\left(m_{2}-1\right)}{\bar{m}} \sum_{k=1}^{2} b_{k}\left(1-\bar{\chi}_{\mathrm{R}}\right)^{k}
\end{array}
$$

where

$$
D\left(\bar{m}, \bar{\chi}_{R}\right)=\left[\delta_{1 m_{1}}-\delta_{1 m_{2}}\right]^{2}\left(d_{1}-\frac{d_{2}}{\bar{m}}\right)\left(1-\bar{\chi}_{R}\right) .
$$

The last equation, with $\delta$ being a Kronecker delta, is a correction factor for the case of a hard sphere and a partially flexible (rod-coil) chain fluid. For any other case, it reduces to zero. The $a_{2}, a_{3}$, and $b_{2}$ parameters have an additional dependence on chain length as

$$
\begin{aligned}
& a_{2}=a_{21}+\frac{a_{22}}{\bar{m}}, \\
& a_{3}=a_{31}+\frac{a_{32}}{\bar{m}}, \\
& b_{2}=b_{21}+\frac{b_{22}}{\bar{m}} .
\end{aligned}
$$


TABLE I. The 10 model constants for the coefficients (Eqs. (C1)-(C7)) of the excluded-volume expression from Eq. (10).

\begin{tabular}{lrrrccc}
\hline \hline $\mathrm{i}$ & $a_{1}$ & $a_{2 i}$ & $a_{3 i}$ & $b_{1}$ & $b_{2 i}$ & $d_{i}$ \\
\hline 1 & 4.63 & -4.71 & 1.31 & 0.305 & -0.171 & 0.125 \\
2 & & 7.84 & -6.18 & & 3.32 & 0.206 \\
\hline
\end{tabular}

Here, $a_{1}, a_{21}, a_{22}, a_{31}, a_{32}, b_{1}, b_{21}$, and $b_{22}$ are dimensionless constants which were adjusted to MC data for the pairexcluded volume of pure rod-coil fluids. The dimensionless constants $d_{1}$ and $d_{2}$ where adjusted to MC data for the pairexcluded volume of a hard sphere and rod-coil fluids. The values of these constants are listed in Table I.

${ }^{1}$ M. Schadt, Annu. Rev. Mater. Sci. 27, 305 (1997).

${ }^{2}$ P. G. de Gennes and J. Prost, The Physics of Liquid Crystals, 2nd ed. (Oxford University Press, Oxford, 1993).

${ }^{3}$ R. Eelkema and B. L. Feringa, Org. Biomol. Chem. 4, 3729 (2006).

${ }^{4}$ N. Tamaoki, Adv. Mater. 13, 1135 (2001).

${ }^{5}$ D. Dutta, A. Fruitwala, A. Kohli, and R. A. Weiss, Polym. Eng. Sci. 30, 1005 (1990)

${ }^{6}$ N. Kapernaum, C. S. Hartley, J. C. Roberts, R. P. Lemieux, and F. Giesselmann, Beilstein J. Org. Chem. 5, 65 (2009).

${ }^{7}$ N. Kapernaum, C. S. Hartley, J. C. Roberts, F. Schoerg, D. Krueerke, R. P. Lemieux, and F. Giesselmann, ChemPhysChem 11, 2099 (2010).

${ }^{8}$ L. Onsager, Phys. Rev. 62, 558 (1942).

${ }^{9}$ L. Onsager, Ann. N. Y. Acad. Sci. 51, 627 (1949).

${ }^{10} \mathrm{~T}$. Itou and A. Tiramoto, Macromolecules 17, 1419 (1984).

${ }^{11}$ T. Itou and A. Tiramoto, Polym. J. (Tokyo, Jpn.) 16, 779 (1984).

${ }^{12}$ P. A. Buining and H. N. W. Lekkerkerker, J. Phys. Chem. 97, 11510 (1993).

${ }^{13}$ P. J. Flory and A. Abe, Macromolecules 11, 1119 (1978).

${ }^{14}$ H. N. W. Lekkerkerker, P. Coulon, R. Van Der Haegen, and R. Deblieck, J. Chem. Phys. 80, 3427 (1984).

${ }^{15}$ T. Odijk and H. N. W. Lekkerkerker, J. Phys. Chem. 89, 2090 (1985).

${ }^{16}$ G. J. Vroege and H. N. W. Lekkerkerker, J. Phys. Chem. 97, 3601 (1993).

${ }^{17}$ S. Varga, A. Galindo, and G. Jackson, J. Chem. Phys. 117, 7207 (2002).

${ }^{18}$ A. Galindo, A. J. Haslam, S. Varga, G. Jackson, and A. G. Vanakaras, J. Chem. Phys. 119, 5216 (2003).

${ }^{19}$ G. Cinacchi, L. Mederos, and E. Velasco, J. Chem. Phys. 121, 3854 (2004).

${ }^{20}$ S. Varga, K. R. Purdy, A. Galindo, S. Fraden, and G. Jackson, Phys. Rev. E 72, 051704 (2005)

${ }^{21}$ G. Cinacchi, Y. Martínez-Ratón, L. Mederos, and E. Velasco, J. Chem. Phys. 124, 234904 (2006)

${ }^{22}$ A. Cuetos, B. Martínez-Haya, S. Lago, and L. F. Rull, Phys. Rev. E 75, 061701 (2007)

${ }^{23}$ J. Philips and M. Schmidt, Phys. Rev. E 81, 041401 (2010).

${ }^{24}$ F. A. Escobedo and J. J. de Pablo, J. Chem. Phys. 106, 9858 (1997).

${ }^{25}$ P. J. Camp and M. P. Allen, Physica A 229, 410 (1996).

${ }^{26}$ P. J. Camp, M. P. Allen, P. G. Bolhuis, and D. Frenkel, J. Chem. Phys. 106, 9270 (1997)

${ }^{27}$ H. H. Wensink, G. J. Vroege, and H. N. W. Lekkerkerker, J. Phys. Chem. B 105, 10610 (2001).

${ }^{28}$ M. Dijkstra and R. van Roij, Phys. Rev. E 56, 5594 (1997).

${ }^{29}$ G. Nounesis, S. Kumar, S. Pfeiffer, R. Shahidhar, and C. W. Garland, Phys. Rev. Lett. 73, 565 (1994).

${ }^{30}$ K. R. Purdy, S. Varga, A. Galindo, G. Jackson, and S. Fraden, Phys. Rev. Lett. 94, 057801 (2005).

${ }^{31}$ F. M. van der Kooij, D. van der Beek, and H. N. W. Lekkerkerker, J. Phys. Chem. B 105, 1696 (2001).

${ }^{32}$ C. Vega and S. Lago, J. Chem. Phys. 100, 6727 (1994).

${ }^{33}$ T. van Westen, B. Oyarzún, T. J. H. Vlugt, and J. Gross, J. Chem. Phys. 139, 034505 (2013).
${ }^{34}$ P. J. Flory, Proc. R. Soc. London, Ser. A 234, 60 (1956).

${ }^{35}$ A. R. Khokhlov, Phys. Lett. A 68, 135 (1978).

${ }^{36}$ A. R. Khokhlov and A. N. Semenov, Physica A 108, 546 (1981).

${ }^{37}$ A. R. Khokhlov and A. N. Semenov, Physica A 112, 605 (1982).

${ }^{38}$ D. B. Dupré and S. Yang, J. Chem. Phys. 94, 7466 (1991).

${ }^{39}$ M. Dijkstra and D. Frenkel, Phys. Rev. E 51, 5891 (1995).

${ }^{40}$ H. Fynewever and A. Yethiraj, J. Chem. Phys. 108, 1636 (1998).

${ }^{41}$ D. C. Williamson and G. Jackson, J. Chem. Phys. 108, 10294 (1998).

${ }^{42}$ K. M. Jaffer, S. B. Opps, and D. E. Sullivan, J. Chem. Phys. 110, 11630 (1999).

${ }^{43}$ T. Hino and J. M. Prausnitz, Polymer 40, 1241 (1999).

${ }^{44}$ K. M. Jaffer, S. B. Opps, D. E. Sullivan, B. G. Nickel, and L. Mederos, J. Chem. Phys. 114, 3314 (2001).

${ }^{45}$ C. Vega, C. McBride, and L. G. MacDowell, J. Chem. Phys. 115, 4203 (2001)

${ }^{46}$ C. McBride, C. Vega, and L. G. MacDowell, Phys. Rev. E 64, 011703 (2001)

${ }^{47}$ C. McBride and C. Vega, J. Chem. Phys. 117, 10370 (2002).

${ }^{48}$ R. Diplock, D. E. Sullivan, K. M. Jaffer, and S. B. Opps, Phys. Rev. E 69, 062701 (2004).

${ }^{49}$ T. Jiang and J. Wu, J. Chem. Phys. 127, 034902 (2007).

${ }^{50}$ T. van Westen, T. J. H. Vlugt, and J. Gross, J. Chem. Phys. 137, 044906 (2012)

${ }^{51}$ G. R. Luckhurst and G. W. Gray, Molecular Physics of Liquid Crystals (Academic Press, London, 1979).

${ }^{52}$ G. W. Gray, Thermotropic Liquid Crystals (John Wiley \& Sons, Chichester, 1987), Chap. 2.

${ }^{53}$ T. van Westen, B. Oyarzún, T. J. H. Vlugt, and J. Gross, Mol. Phys. (published online).

${ }^{54}$ M. Franco-Melgar, A. J. Haslam, and G. Jackson, Mol. Phys. 106, 649 (2008).

${ }^{55}$ M. S. Wertheim, J. Stat. Phys. 42, 477 (1986).

${ }^{56} \mathrm{H}$. Liu and Y. Hu, Fluid Phase Equilib. 122, 75 (1996)

${ }^{57}$ Y. Hu, H. Liu, and J. M. Prausnitz, J. Chem. Phys. 104, 396 (1996).

${ }^{58}$ J. E. Mayer and M. G. Mayer, Statistical Mechanics, 7th ed. (John Wiley \& Sons, New York, 1940), Chap. 13

${ }^{59}$ S. I. Sandler, An Introduction to Applied Statistical Thermodynamics (Wiley, Hoboken, 2011)

${ }^{60}$ D. C. Williamson and G. Jackson, Mol. Phys. 86, 819 (1995).

${ }^{61}$ A. Malijevsky, G. Jackson, and S. Varga, J. Chem. Phys. 129, 144504 (2008).

${ }^{62}$ W. H. Press, S. A. Teukolsky, W. T. Vetterling, and B. P. Flannery, Numerical Recipes in Fortran 77 (Cambridge University Press, Cambridge, 1992).

${ }^{63}$ M. Franco-Melgar, A. J. Haslam, and G. Jackson, Mol. Phys. 110, 3107 (2012).

${ }^{64}$ S. I. Sandler, Phase Equilibria in Chemical Engineering (Butterworth Publishers, Stoneham, 1985).

${ }^{65}$ B. Oyarzún, T. van Westen, and T. J. H. Vlugt, J. Chem. Phys. 138, 204905 (2013).

${ }^{66} \mathrm{~J}$. Gross and P. Jansens, patent application Wo2008147181-a1/nl2000654c2 (2008).

${ }^{67}$ M. de Groen, T. J. H. Vlugt, and T. W. de Loos, J. Phys. Chem. B 116, 9101 (2012).

${ }^{68}$ S. I. Sandler, Chemical, Biochemical, and Engineering Thermodynamics, 4th ed. (Wiley, Hoboken, 2006).

${ }^{69}$ T. Bose, C. D. Mukherjee, M. K. Roy, and M. Saha, Mol. Cryst. Liq. Cryst. 126, 197 (1985)

${ }^{70}$ N. V. Madhusudana and J. Rajan, Liq. Cryst. 7, 31 (1990).

${ }^{71}$ A. Prasad and M. K. Das, Phase Transitions 82, 780 (2009).

${ }^{72}$ A. Prasad and M. K. Das, Phase Transitions 83, 1072 (2010).

${ }^{73}$ N. Kapernaum, F. Knecht, C. S. Hartley, J. C. Roberts, R. P. Lemieux, and F. Giesselmann, Beilstein J. Org. Chem. 8, 1118 (2012).

${ }^{74}$ J. P. Hansen and I. R. McDonald, Theory of Simple Liquids, 3rd ed. (Academic Press, London, 2006) 